\title{
MAJORIZATION IN DE BRANGES SPACES. III. DIVISION BY BLASCHKE PRODUCTS
}

\author{
A. BARANOV AND H. WORACEK
}

Dedicated to Victor Petrovich Havin on the occasion of his 75th birthday

\begin{abstract}
This paper is a part of a series dealing with subspaces of de Branges spaces of entire functions generated by majorization on subsets of the closed upper half-plane. In the present, third, part the study of a certain Banach space generated by an admissible majorant is continued. The main theme is "invariance of the unit ball with respect to division by Blaschke products". In connection with this topic, representability via special types of majorants plays an important role. Some (positive and negative) results on invariance under division by Blaschke factors are obtained, and the unit balls representable by log-superharmonic majorants are characterized.
\end{abstract}

\section{§1. INTRODUCTION}

A de Branges space $\mathcal{H}$ is a Hilbert space of entire functions that satisfies the following axioms.

(dB1) For each $w \in \mathbb{C}$ the point evaluation functional $F \mapsto F(w)$ is continuous in the norm of $\mathcal{H}$.

(dB2) If $F \in \mathcal{H}$, the function $F^{\#}(z):=\overline{F(\bar{z})}$ also belongs to $\mathcal{H}$ and $\left\|F^{\#}\right\|_{\mathcal{H}}=\|F\|_{\mathcal{H}}$.

(dB3) If $w \in \mathbb{C} \backslash \mathbb{R}$ and $F \in \mathcal{H}, F(w)=0$, then

$$
\frac{z-\bar{w}}{z-w} F(z) \in \mathcal{H} \quad \text { and } \quad\left\|\frac{z-\bar{w}}{z-w} F(z)\right\|_{\mathcal{H}}=\|F\|_{\mathcal{H}} .
$$

In dB1- dB5, L. de Branges developed a deep and rich structure theory of such spaces. The key role there was played by the de Branges subspaces of a given space $\mathcal{H}$, i.e., the subspaces of $\mathcal{H}$ that become themselves de Branges spaces if endowed with the inner product inherited from $\mathcal{H}$.

Originating from the Beurling-Malliavin multiplier theorem, and some more recent generalizations to shift-coinvariant subspaces of the Hardy space, cf. [BM], HM1, HM2, a general concept of generating de Branges subspaces by majorization has evolved: If $\mathfrak{m}$ is a function defined on some subset $D$ of the closed upper half-plane $\mathbb{C}^{+} \cup \mathbb{R}$, one may define

$$
\mathcal{R}_{\mathfrak{m}}(\mathcal{H}):=\operatorname{clos}_{\mathcal{H}}\{F \in \mathcal{H}: \exists C>0:|F(z)|,|F(\bar{z})| \leq C \mathfrak{m}(z), z \in D\} .
$$

Here $\operatorname{clos} \mathcal{H}$ stands for the closure taken in the norm of $\mathcal{H}$. Provided that $\mathcal{R}_{\mathfrak{m}}(\mathcal{H}) \neq\{0\}$ and $\mathfrak{m}$ satisfies some mild regularity condition, $\mathcal{R}_{\mathfrak{m}}(\mathcal{H})$ is a de Branges subspace of $\mathcal{H}$. We have studied this concept in our previous papers BW1 - BW4]. For example, we showed that every de Branges subspace of $\mathcal{H}$ can be obtained in this way and investigated which

2010 Mathematics Subject Classification. Primary 46E15, 46E22, 30J10.

Key words and phrases. de Branges subspace, majorant, subharmonic function, Blaschke product. 
majorants defined on which subsets produce (or may produce) a prescribed (family of) de Branges subspaces. Also, we considered the linear space

$$
R_{\mathfrak{m}}(\mathcal{H}):=\{F \in \mathcal{H}: \exists C>0:|F(z)|,|F(\bar{z})| \leq C \mathfrak{m}(z), z \in D\},
$$

which becomes a Banach space if endowed with a stronger norm, namely,

$$
\begin{aligned}
& \|F\|_{\mathfrak{m}}:=\max \left\{\|F\|_{\mathcal{H}}, \min \left\{C \geq 0:|F(z)|,\left|F^{\#}(z)\right| \leq C \mathfrak{m}(z), z \in D\right\}\right\}, \\
& F \in R_{\mathfrak{m}}(\mathcal{H}) .
\end{aligned}
$$

Apparently, the space $R_{\mathfrak{m}}(\mathcal{H})$ is exactly that part of $\mathcal{R}_{\mathfrak{m}}(\mathcal{H})$ about whose elements one has explicit information. Hence, this Banach space is an object of interest. In general, the geometric structure of $R_{\mathfrak{m}}(\mathcal{H})$ is complicated, but an intriguing topic. For example, in most cases, $R_{\mathfrak{m}}(\mathcal{H})$ will not be reflexive; cf. [BW4.

The present paper is devoted to a further study of $R_{\mathfrak{m}}(\mathcal{H})$. It is more oriented towards complex analysis topics than towards Banach space questions; the main theme here is "dividing out zeros" and "subharmonicity".

Now we give a short overview of the main results of the paper, sometimes in a simplified and less general form (in brackets we refer to the corresponding statements in the text).

It is well known that if $\mathcal{H}$ is a de Branges space and $\mathcal{L}$ is one of its de Branges subspaces, then the unit ball

$$
B(\mathcal{L}):=\left\{F \in \mathcal{L}:\|F\|_{\mathcal{H}} \leq 1\right\}
$$

of $\mathcal{L}$ is invariant with respect to dividing out zeros in the following sense: If $F \in B(\mathcal{L})$ and $P$ is a Blaschke product for $\mathbb{C}^{+}$such that $P^{-1} F$ is entire, then $P^{-1} F \in B(\mathcal{L})$. We pose the question whether the same holds true for the unit ball

$$
B_{\mathfrak{m}}(\mathcal{H}):=\left\{F \in R_{\mathfrak{m}}(\mathcal{H}):\|F\|_{\mathfrak{m}} \leq 1\right\}
$$

of the Banach space $R_{\mathfrak{m}}(\mathcal{H})$. In general, the answer will be negative; obstructions are naturally appearing when majorization is required off the real axis (see, e.g., Example 4.2). However, for a particular class of majorants $\mathfrak{m}$ defined on open subsets of $\mathbb{C}^{+}$, positive answers can be given. The crucial property of $\mathfrak{m}$ in this respect is that the function $-\log \mathfrak{m}$ is subharmonic; we will call such majorants $\log$-superharmonic. This property allows us to employ the Phragmén-Lindelöf principle.

Theorem 1.1 (Theorem 4.4 and Corollary 4.5). Let $D$ be an open subset of $\mathbb{C}^{+}$, and let $\mathfrak{m} \in \operatorname{Adm}_{D} \mathcal{H}$ be log-superharmonic. Moreover, let $F \in B_{\mathfrak{m}}(\mathcal{H}) \backslash\{0\}$, and let $P$ be a Blaschke product for $\mathbb{C}^{+}$with $\left.\mathfrak{d}_{P}\right|_{\mathbb{C}^{+}} \leq\left.\mathfrak{d}_{F}\right|_{\mathbb{C}^{+}}$. If, for some $\beta>0$,

$$
\left|\frac{F(\zeta)}{P(\zeta)}\right| \leq \beta \liminf _{\substack{z \rightarrow \zeta \\ z \in D}} \mathfrak{m}(z), \quad \zeta \in \partial D \backslash \mathbb{R},
$$

then $P^{-1} F \in R_{\mathfrak{m}}(\mathcal{H})$, and

$$
\left|\frac{F(z)}{P(z)}\right| \leq \max \left\{\beta,\|F\|_{\mathfrak{m}}\right\} \mathfrak{m}(z), \quad z \in D .
$$

In particular, if $\mathfrak{m} \in \operatorname{Adm}_{\mathbb{C}^{+}} \mathcal{H}$ is log-superharmonic, $F \in B_{\mathfrak{m}}(\mathcal{H})$, and $P$ is a Blaschke product for $\mathbb{C}^{+}$with $\left.\mathfrak{d}_{P}\right|_{\mathbb{C}^{+}} \leq\left.\mathfrak{d}_{F}\right|_{\mathbb{C}^{+}}$, then $P^{-1} F \in B_{\mathfrak{m}}(\mathcal{H})$.

Of course, being log-superharmonic is quite a strong property. However, it should be noted that every de Branges subspace of $\mathcal{H}$ can still be realized as $\mathcal{R}_{\mathfrak{m}}(\mathcal{H})$ with a majorant $\mathfrak{m}$ of this kind; cf. BW3. In connection with the division problem, the following question arises: Which unit balls $B_{\mathfrak{m}_{0}}(\mathcal{H})$ (or, more generally, which subsets $B$ of $\mathcal{H}$ ) are equal to a unit ball $B_{\mathfrak{m}}(\mathcal{H})$ with some log-superharmonic majorant $\mathfrak{m}$ defined on all of $\mathbb{C}^{+}$? 
We also consider two more special classes of majorants, namely, Smirnov class $\mathcal{N}_{+}$ and Hardy class $H^{p}$ majorants. A function $\mathfrak{m} \in \operatorname{Adm}_{\mathbb{C}^{+}} \mathcal{H}$ is called an $\mathcal{N}_{+}$-majorant (an $H^{p}$-majorant, $\left.p>0\right)$ for $\mathcal{H}$ if it is of the form

$$
\mathfrak{m}(z)=\left|e^{-i a z} f(z) E(z)\right|, \quad z \in \mathbb{C}^{+},
$$

with some $a \leq 0$ and $f$ being outer (respectively, $f$ being an outer function in $H^{p}$ ).

The next theorem gives a description of the subsets $B$ that can be realized as unit balls with some log-superharmonic majorant in $\mathbb{C}^{+}$. Interestingly, it turns out that these balls can already be realized by majorization along the real axis in conjunction with a restriction of exponential growth; also, a majorant can always be chosen to be an $\mathcal{N}_{+}$-majorant. In the statement we use the upper envelope majorant $\mathfrak{m}_{B}(w)=\sup _{F \in B}|F(w)|$.

Theorem 1.2 (Theorem 5.3). Let $\mathcal{H}$ be a de Branges space, and let $E \in \mathcal{H} B$ be such that $\mathcal{H}=\mathcal{H}(E)$. Moreover, let $B \subseteq B(\mathcal{H}), B \neq \varnothing,\{0\}$. Then the following statements are equivalent.

(i) There exists a log-superharmonic majorant $\mathfrak{m} \in \operatorname{Adm}_{\mathbb{C}^{+}} \mathcal{H}$ such that $B=B_{\mathfrak{m}}(\mathcal{H})$.

(ii) There exists an $\mathcal{N}_{+}$-majorant $\mathfrak{m} \in \operatorname{Adm}_{\mathbb{C}^{+}} \mathcal{H}$ such that $B=B_{\mathfrak{m}}(\mathcal{H})$.

(iii) We have $B=B_{\mathfrak{m}_{B} \mid \mathbb{R}}\left(\mathcal{H}_{(a)}\right)$ (majorization on the real axis) and

$$
\int_{D}\left(\log +\frac{\mathfrak{m}_{B}(t)}{|E(t)|}\right) \frac{d t}{1+t^{2}}<\infty
$$

Here $a=\mathrm{mt}_{\mathcal{H}} B$ is the so-called mean type of the set $B$, and

$$
\mathcal{H}_{(a)}:=\left\{F \in \mathcal{H}: \operatorname{mt}_{\mathcal{H}} F, \operatorname{mt}_{\mathcal{H}} F^{\#} \leq a\right\}
$$

is the subspace defined by exponential growth.

The convergence of the logarithmic integral in (1.1) is a mild restriction; it is satisfied, e.g., whenever the function $E$ is of finite order (see Proposition 5.10).

A similar description can be given for sets $B$ representable as unit balls $B_{\mathfrak{m}}(\mathcal{H})$ with some $H^{p}$-majorant $\mathfrak{m}$.

Proposition 1.3 (Proposition 5.14$)$. Let $\mathcal{H}$ be a de Branges space, let $B$ be a nonempty subset of its unit ball, and let $p \in(0, \infty)$. Then the following statements are equivalent:

(i) there exists an $H^{p}$-majorant $\mathfrak{m} \in \operatorname{Adm}_{\mathbb{C}^{+}} \mathcal{H}$ such that $B=B_{\mathfrak{m}}(\mathcal{H})$;

(ii) we have $B=B_{\left.\mathfrak{m}_{B}\right|_{\mathbb{R}}}\left(\mathcal{H}_{(a)}\right), a=\operatorname{mt}_{\mathcal{H}} B$, and $\int_{\mathbb{R}}\left(\frac{\mathfrak{m}_{B}(t)}{|E(t)|}\right)^{p} d t<\infty$.

In the last part of the paper we study the question whether a unit ball $B_{\mathfrak{m}_{0}}(\mathcal{H})$ generated by an arbitrary majorant is contained in or contains unit balls $B_{\mathfrak{m}}(\mathcal{H})$ generated by log-superharmonic majorants.

This question is also of interest for the following reason. In the first case, we obtain supersets $B$ such that division of a function $F$ in $B_{\mathfrak{m}_{0}}(\mathcal{H})$ by a Blaschke product cannot lead further out than $B$ and, in the second case, we obtain subsets $B$ which are invariant with respect to division by Blaschke products.

It is a noteworthy fact that the description of the balls that are larger than $B_{\mathfrak{m}_{0}}(\mathcal{H})$ is fairly simple, whereas it is quite hard to get a handle on the set of those contained in $B_{\mathfrak{m}_{0}}(\mathcal{H})$. The following theorem provides an answer to the first question. Here we denote by $\mathfrak{m}_{0}^{b}$ the upper envelope, $\mathfrak{m}_{0}^{b}(w)=\sup _{F \in B_{\mathfrak{m}_{0}}(\mathcal{H})}|F(w)|, w \in \mathbb{C}^{+}$.

Theorem 1.4 (Theorem 6.3). Let $\mathcal{H}=\mathcal{H}(E)$ be a de Branges space, and let $\mathfrak{m}_{0} \in$ $\operatorname{Adm} \mathcal{H}$. Then a log-superharmonic majorant $\mathfrak{m}$ with $B_{\mathfrak{m}_{0}}(\mathcal{H}) \subseteq B_{\mathfrak{m}}(\mathcal{H})$ exists if and only if

$$
\int_{\mathbb{R}}\left(\log ^{+} \frac{\mathfrak{m}_{0}^{b}(t)}{|E(t)|}\right) \frac{d t}{1+t^{2}}<\infty .
$$


The problem of existence of smaller balls generated by log-superharmonic majorants is subtler and is related to a completely different topic, namely to the existence of real zero-free elements in $B_{\mathfrak{m}_{0}}(\mathcal{H})$.

Theorem 1.5 (Theorem 6.5). Let $\mathcal{H}$ be a de Branges space, and let $\mathfrak{m}_{0} \in \operatorname{Adm} \mathcal{H}$. Then the following statements are equivalent:

(i) there exists an $H^{2}$-majorant $\mathfrak{m}$ with $B_{\mathfrak{m}}(\mathcal{H}) \subseteq B_{\mathfrak{m}_{0}}(\mathcal{H})$;

(ii) there exists an element $F \in B_{\mathfrak{m}_{0}}(\mathcal{H})$ that satisfies $F^{\#}=F$ and has no zeros in $\mathbb{C} \backslash \mathbb{R}$

The paper is organized as follows. $\S 2$ and $\S 3$ are of a preliminary character. In $\S 2$ we set up our notation, recall some basic facts on de Branges spaces, and provide some preparatory results, among them a version of the monotone convergence theorem for nondecreasing nets (rather than sequences) of functions. In $\S 3$ we introduce the majorants $\mathfrak{m}_{B}$ and $\mathfrak{m}_{B}^{\perp}$ associated with a subset $B \subseteq B(\mathcal{H})$. These are essential tools, and they will be extensively used throughout the paper.

$\S 4$ and $\S 5$ contain the main results of the paper. In $\S 4$ we study the problem of division by Blaschke products and prove Theorem 4.4 on dividing out zeros for log-superharmonic majorants. In $\S 5$ we characterize the subsets $B$ of a de Branges space $\mathcal{H}$ that are equal to a unit ball $B_{\mathfrak{m}}(\mathcal{H})$ with some $\log$-superharmonic majorant defined on all of $\mathbb{C}^{+}$. Finally, in $\S 6$, we study the question as to whether a unit ball $B_{\mathfrak{m}_{0}}(\mathcal{H})$ generated by an arbitrary majorant is contained in or contains unit balls $B_{\mathfrak{m}}(\mathcal{H})$ generated by log-superharmonic majorants.

\section{§2. Preliminaries}

a. Functions of bounded type. To start with, we recall some notation from the bounded type theory. Our standard reference in this respect will be RR.

We shall denote by $\mathcal{N}\left(\mathbb{C}^{+}\right)$the class of functions that are analytic and of bounded type in $\mathbb{C}^{+}$, and by $H^{p}\left(\mathbb{C}^{+}\right), p \in(0, \infty)$, the respective Hardy space. Moreover, $\mathcal{N}_{+}\left(\mathbb{C}^{+}\right)$will denote the Smirnov class, consisting of all functions $f$ of bounded type whose inner-outer factorization is of the form $B S F$ with a Blaschke product $B$, a singular inner function $S$, and an outer function $F$. Since we use this notation exclusively for the open upper half-plane, we shall drop the argument $\mathbb{C}^{+}$.

For later use, we recall the definition of an outer function. If $k: \mathbb{R} \rightarrow[0, \infty]$ is measurable and $\log k \in L^{1}\left(\frac{d t}{1+t^{2}}\right)$, then an analytic function $\mathfrak{f}_{k}$ is well defined on $\mathbb{C}^{+}$by

$$
\mathfrak{f}_{k}(z):=\exp \left(\frac{1}{i \pi} \int_{\mathbb{R}}\left(\frac{1}{t-z}-\frac{t}{1+t^{2}}\right) \log k(t) d t\right) .
$$

The boundary values of $\left|\mathfrak{f}_{k}\right|$ along $\mathbb{R}$ are equal almost everywhere to $k$. Actually, the function $\log \left|\mathfrak{f}_{k}\right|$ is simply the Poisson integral of $\log k$. Let us note explicitly that always $\mathfrak{f}_{k_{1}} \cdot \mathfrak{f}_{k_{2}}=\mathfrak{f}_{k_{1} \cdot k_{2}}$.

A function $f$ is said to be outer if it is of the form $\gamma \mathfrak{f}_{k}$ with some $\gamma \in \mathbb{C},|\gamma|=1$, and some $k: \mathbb{R} \rightarrow[0, \infty], \log k \in L^{1}\left(\frac{d t}{1+t^{2}}\right)$. Sometimes, one speaks more specifically of an outer function for $\mathcal{N}$. If, additionally, the function $k$ belongs to $L^{p}(d t)$, then $f$ belongs to $H^{p}$, and one says that $f$ is outer for $H^{p}$.

The following statement will be used later on. It is checked by a simple and standard argument and, apparently, is well known. Therefore, we omit the proof.

Lemma 2.1. Let $k: \mathbb{R} \rightarrow[0, \infty]$ be such that $\log k \in L^{1}\left(\frac{d t}{1+t^{2}}\right)$. Assume that

(i) $k$ is continuous;

(ii) the set $S:=k^{-1}(\{0, \infty\})$ is discrete; 
(iii) for each $x_{0} \in S$ there exists a number $n\left(x_{0}\right) \in \mathbb{Z}$ such that

$$
\lim _{t \rightarrow x_{0}} \frac{k(t)}{\left|t-x_{0}\right|^{n\left(x_{0}\right)}} \in(0, \infty)
$$

Let $F$ be a function defined and meromorphic on some domain $G \supseteq \mathbb{C}^{+} \cup \mathbb{R}$ and satisfying

$$
\lim _{z \rightarrow x_{0}} \frac{|F(z)|}{\left|z-x_{0}\right|^{n\left(x_{0}\right)}} \in(0, \infty), x_{0} \in S, \quad F(z) \neq 0, \infty, \quad z \in G \backslash S .
$$

Then the function $\left|F^{-1} \mathfrak{f}_{k}\right|$ has a continuous and positive extension to $\mathbb{C}^{+} \cup \mathbb{R}$, namely, by

$$
\begin{cases}\frac{k(x)}{|F(x)|}, & x \notin S \\ \lim _{t \rightarrow x_{0}} \frac{k(t)}{|F(t)|}, & x \in S\end{cases}
$$

b. Zero-divisors, mean type, ordering. First, we deal with zero-divisors associated with a function. Let $D \subseteq \mathbb{C}$ be a nonempty open set, and let $f: D \rightarrow \mathbb{C}$ be analytic and not identically zero. Then the zero-divisor $\mathfrak{d}_{f}$ associated with $f$ is the map that assigns to each point $w \in D$ the multiplicity of $w$ as a zero of $f$. In other words, $\mathfrak{d}_{f}(w)$ is a unique nonnegative integer such that

$$
\lim _{z \rightarrow w} \frac{|f(z)|}{|z-w|^{\mathfrak{d}_{f}(w)}} \in(0, \infty) .
$$

We shall use a slightly different formulation of this definition, in order to be able to apply the notation $\mathfrak{d}_{f}$ to arbitrary functions.

Definition 2.2. Let $D \subseteq \mathbb{C}$, and let $f: D \rightarrow \mathbb{C}$ be an arbitrary function. Then the zero-divisor $\mathfrak{d}_{f}$ of $f$ is the function $\mathfrak{d}_{f}: \mathbb{C} \rightarrow \mathbb{N}_{0} \cup\{+\infty\}$ defined as

$$
\mathfrak{d}_{f}(w):=\inf \left\{n \in \mathbb{N}_{0}: \begin{array}{l}
\exists \text { neighborhood } U \text { of } w \text { such that } \\
\inf _{|z-w|^{n} \neq 0}|z-w|^{-n}|f(z)|>0
\end{array}\right\}, \quad w \in \mathbb{C} .
$$

Here the infimum of the empty set is defined to be $+\infty$.

For analytic functions $f$ and points $w \in D$, this definition of $\mathfrak{d}_{f}(w)$ clearly coincides with the usual definition via (2.2). Of course, for an arbitrary function $f$ we have no limit relation such as (2.2). But at least the rate of decay of $f$ towards $w$ will be bounded by $|z-w|^{\mathfrak{d}_{f}(w)}$. Moreover, we note that the above definition of $\mathfrak{d}_{f}$ is made in such a way that $\mathfrak{d}_{f}(w)=0, w \notin \bar{D}$.

The notation $\mathfrak{d}_{\text {index }}$ will also be applied when index is not a single function, but a set of functions: if $B$ is a set of functions, we denote

$$
\mathfrak{d}_{B}(w):=\inf \left\{\mathfrak{d}_{f}(w): f \in B\right\} .
$$

Note that, provided $B$ is nonempty, this infimum is attained.

Next, we discuss the notion of the mean type. If $f$ is analytic and of bounded type in $\mathbb{C}^{+}$, then there exists a number $c \in \mathbb{R}$ such that

$$
\limsup _{\substack{r \rightarrow \infty \\ r \in M}} \frac{1}{r} \log \left|f\left(a+r e^{i \theta}\right)\right|=c \cdot \sin \theta
$$

whenever $\theta \in(0, \pi), a \in \mathbb{R}$, and $M \subseteq \mathbb{R}^{+}$is a subset of infinite logarithmic length. The number $c$ is usually referred to as the mean type of $f$. Again, we wish to apply the notion of mean type to a broader class of functions. 
Definition 2.3. Let $D \subseteq \mathbb{C}^{+}$, and let $f: D \rightarrow \mathbb{C}$ be an arbitrary function. Then the mean type of $f$ is defined as the number

$$
\operatorname{mt} f:=\inf \left\{\frac{1}{\sin \theta} \limsup _{\substack{r \rightarrow \infty \\ r \in M}} \frac{1}{r} \log \left|f\left(a+r e^{i \theta}\right)\right|\right\} \in[-\infty,+\infty],
$$

where the infimum is taken over all $\theta \in(0, \pi), a \in \mathbb{R}$, and subsets $M \subseteq \mathbb{R}^{+}$of infinite logarithmic length such that $\left\{a+r e^{i \theta}: r \in M\right\} \subseteq D$. If $B$ is a set of functions, we set $\operatorname{mt} B:=\sup \{\operatorname{mt} f: f \in B\}$.

Apparently, this notion coincides with the usual one if $f \in \mathcal{N}$. Of course, for an arbitrary function $f$, we cannot expect a regular growth behavior such as (2.3). But at least the asymptotic growth of $f$ on some not too small set is controlled by mt $f$.

Finally, we define a partial order on functions taking nonnegative values.

Definition 2.4. Let $D_{i} \subseteq \mathbb{C}$, and let $f_{i}: D_{i} \rightarrow[0, \infty), i=1,2$. Then we shall write

$$
f_{1} \preccurlyeq f_{2} \stackrel{\text { def }}{\Longleftrightarrow} D_{1} \supseteq D_{2} \text { and }\left.f_{1}\right|_{D_{2}} \leq f_{2} .
$$

In the context of majorization, the use of this notation is sometimes practical. Let us explain this fact. Denote by $\operatorname{Adm} \mathcal{H}$ the set of all functions $\mathfrak{m}: D \rightarrow[0, \infty]$, where $D$ is some nonempty subset of $\mathbb{C}^{+} \cup \mathbb{R}$, such that

(Adm1) $\quad \operatorname{supp} \mathfrak{d}_{\mathfrak{m}} \subseteq \mathbb{R}$;

$(\operatorname{Adm2}) \quad R_{\mathfrak{m}}(\mathcal{H})$ contains a function that does not vanish identically.

If we wish to be specific about the domain of $\mathfrak{m}$, we shall write $\mathfrak{m} \in \operatorname{Adm}_{D} \mathcal{H}$ when $\mathfrak{m}$ is defined on $D$. As we saw in [BW3, Theorem 3.1], these functions are exactly those for which $\mathcal{R}_{\mathfrak{m}}(\mathcal{H})$ becomes a de Branges subspace of $\mathcal{H}$.

Next, denote by $\beta$ the assignment

$$
\beta:\left\{\begin{aligned}
\operatorname{Adm} \mathcal{H} & \rightarrow\{B \subseteq \mathcal{H}: B \neq \varnothing,\{0\}\}, \\
\mathfrak{m} & \mapsto B_{\mathfrak{m}}(\mathcal{H}) .
\end{aligned}\right.
$$

The relevance of the relation "ß" is seen from the following simple observation: The assignment $\beta$ is order-preserving, i.e., $\mathfrak{m}_{1} \preccurlyeq \mathfrak{m}_{2}$ implies $\beta\left(\mathfrak{m}_{1}\right) \subseteq \beta\left(\mathfrak{m}_{2}\right)$.

c. De Branges spaces. Most of the facts collected in this subsection can be found in dB6. To start with, note that, by (dB1), the space $\mathcal{H}$ is a reproducing kernel Hilbert space. We denote its reproducing kernel by $K(w, z)$; i.e., let $K(w, \cdot)$ be a unique element of $\mathcal{H}$ such that

Moreover, we set

$$
F(w):=(F, K(w, \cdot))_{\mathcal{H}}, \quad F \in \mathcal{H}, w \in \mathbb{C} .
$$

$$
\nabla_{\mathcal{H}}(w):=\|K(w, \cdot)\|_{\mathcal{H}}=K(w, w)^{\frac{1}{2}}, \quad w \in \mathbb{C} .
$$

The function $w \mapsto K(w, \cdot)$ is a (weakly- and hence norm-) analytic Banach space-valued function. In particular, this implies that the function $\nabla_{\mathcal{H}}$ is continuous. Moreover, note that, by (dB2), we have $\nabla_{\mathcal{H}}(\bar{z})=\nabla_{\mathcal{H}}(z), z \in \mathbb{C}$. By (dB3), the function $\nabla_{\mathcal{H}}$ does not vanish at any point of $\mathbb{C} \backslash \mathbb{R}$.

At the very beginning of our exposition we gave an axiomatic definition of de Branges spaces, namely, via (dB1)-(dB3). For many purposes it is essential that the de Branges spaces can be constructed also in a more specific way.

Definition 2.5. An entire function $E$ is said to be of Hermite-Biehler class if it satisfies

(HB) $\quad|E(\bar{z})|<|E(z)|, \quad z \in \mathbb{C}^{+}$.

The set of all Hermite-Biehler functions is denoted by $\mathcal{H B}$. 
For each function $E \in \mathcal{H} B$, there exists a continuous and monotone increasing function $\varphi_{E}: \mathbb{R} \rightarrow \mathbb{R}$ such that

$$
E(x)=|E(x)| \exp \left(-i \varphi_{E}(x)\right), \quad x \in \mathbb{R} .
$$

A function $\varphi_{E}$ with these properties is called a phase function of $E$. Clearly, every two phase functions of $E$ differ only by an additive constant.

Sometimes, it is also useful to relate the above notions to the zeros of the function $E$. We mention some facts in this direction.

Remark 2.6. Let $E \in \mathcal{H} B$, and denote by $\left(z_{n}\right)_{n}$ the (finite or infinite) sequence of nonreal zeros of $E$.

(i) The sequence $\left(z_{n}\right)_{n}$ satisfies the Blaschke condition

$$
\sum_{n} \operatorname{Im} \frac{1}{z_{n}}<\infty
$$

and we have

$$
\frac{E^{\#}(z)}{E(z)}=\gamma e^{-i a z} \prod_{n} \frac{1-z / \overline{z_{n}}}{1-z / z_{n}}
$$

where $|\gamma|=1$ and $a=\operatorname{mt}\left(E^{-1} E^{\#}\right) \leq 0$.

(ii) We have

$$
\varphi_{E}^{\prime}(x)=-\frac{a}{2}+\sum_{n} \frac{\left|\operatorname{Im} z_{n}\right|}{\left|x-z_{n}\right|^{2}}, \quad x \in \mathbb{R} .
$$

With a function $E \in \mathcal{H} B$, a space of entire functions can be associated.

Definition 2.7. For $E \in \mathcal{H} B$, denote by $\mathcal{H}(E)$ and $\|\cdot\|_{E}$ the linear space and norm:

$$
\begin{aligned}
\mathcal{H}(E) & :=\left\{F \text { entire }: \frac{F}{E}, \frac{F^{\#}}{E} \in H^{2}\right\}, \\
\|F\|_{E} & :=\left(\int_{\mathbb{R}}\left|\frac{F(t)}{E(t)}\right|^{2} d t\right)^{\frac{1}{2}}, \quad F \in \mathcal{H}(E) .
\end{aligned}
$$

2.8. De Branges spaces via Hermite-Biehler functions. The following statements hold true.

(i) If $E \in \mathcal{H B}$, then $\mathcal{H}(E)$ endowed with the norm $\|\cdot\|_{E}$ is a de Branges space.

(ii) If $\mathcal{H}$ is a de Branges space, then there exists a function $E \in \mathcal{H} B$ such that $\mathcal{H}=$ $\mathcal{H}(E)$ and $\|\cdot\|_{\mathcal{H}}=\|\cdot\|_{E}$.

(iii) Let $E_{1}, E_{2} \in \mathcal{H B}$. Then $\mathcal{H}\left(E_{1}\right)=\mathcal{H}\left(E_{2}\right)$ and $\|\cdot\|_{E_{1}}=\|\cdot\|_{E_{2}}$ if and only if there exists a matrix $U \in \mathbb{R}^{2 \times 2}$ with $\operatorname{det} U=1$ such that

$$
\left(A_{2}, B_{2}\right)=\left(A_{1}, B_{1}\right) U
$$

where $A:=\frac{1}{2}\left(E+E^{\#}\right), B:=\frac{i}{2}\left(E-E^{\#}\right)$.

In what follows we shall always understand equality of de Branges spaces as including equality of norms; i.e., writing $\mathcal{H}\left(E_{1}\right)=\mathcal{H}\left(E_{2}\right)$ implicitly includes $\|\cdot\|_{E_{1}}=\|\cdot\|_{E_{2}}$.

Since a de Branges space $\mathcal{H}=\mathcal{H}(E)$ is fully determined by the function $E$, all its properties must correspond to properties of $E$. We mention a couple of relations of that kind.

2.9. Relationship between $\mathcal{H}(E)$ and $E$. Let $\mathcal{H}$ be a de Branges space, and let $E \in \mathcal{H} B$ be such that $\mathcal{H}=\mathcal{H}(E)$. Then:

(i) we have $\mathfrak{d}_{\mathcal{H}}(x)=\mathfrak{d}_{E}(x), x \in \mathbb{R}$; 
(ii) the reproducing kernel $K(w, \cdot)$ of $\mathcal{H}$ is given by

$$
K(w, z)=\frac{E(z) E^{\#}(\bar{w})-E(\bar{w}) E^{\#}(z)}{2 \pi i(\bar{w}-z)}, \quad z, w \in \mathbb{C}, \quad z \neq \bar{w},
$$

and

$$
K(\bar{z}, z):=\frac{i}{2 \pi}\left(\frac{\partial E}{\partial z}(z) E^{\#}(z)-E(z) \frac{\partial E^{\#}}{\partial z}(z)\right), \quad z \in \mathbb{C} ;
$$

(iii) we have

$$
\nabla_{\mathcal{H}}(z)=\left(\frac{|E(z)|^{2}-|E(\bar{z})|^{2}}{4 \pi \operatorname{Im} z}\right)^{1 / 2}, \quad z \in \mathbb{C} \backslash \mathbb{R},
$$

and

$$
\nabla_{\mathcal{H}}(x)=\pi^{-1 / 2}|E(x)|\left(\varphi_{E}^{\prime}(x)\right)^{1 / 2}, \quad x \in \mathbb{R} .
$$

The following statement is very easy to check, yet is still often useful.

Lemma 2.10. Let $\mathcal{H}$ be a de Branges space, and let $E \in \mathcal{H} B$ be such that $\mathcal{H}=\mathcal{H}(E)$. Then:

(i) the function $|E(z)|^{-1} \nabla_{\mathcal{H}}(z), z \in \mathbb{C}^{+}$, has a continuous and positive extension to $\mathbb{C}^{+} \cup \mathbb{R}$

(ii) we have $\operatorname{mt}\left(|E|^{-1} \nabla_{\mathcal{H}}\right)=0$; more precisely,

$$
\lim _{r \rightarrow \infty} \frac{1}{r} \log \frac{\nabla_{\mathcal{H}}\left(r e^{i \vartheta}\right)}{\left|E\left(r e^{i \vartheta}\right)\right|}=0, \quad \vartheta \in(0, \pi) .
$$

Proof. If $E$ has no real zeros, the assertion in (i) is clear. The general case can easily be reduced to this case by dividing out the real zeros of $E$.

Assertion (ii) follows from the inequalities $\left(w_{0} \in \mathbb{C}^{+}\right.$is fixed)

$$
\frac{\left|E\left(w_{0}\right)\right|\left(1-\left|\frac{E\left(\overline{w_{0}}\right)}{E\left(w_{0}\right)}\right|\right)}{2 \pi \nabla_{\mathcal{H}}\left(w_{0}\right)} \frac{1}{\left|z-\bar{w}_{0}\right|} \leq \frac{\nabla_{\mathcal{H}}(z)}{|E(z)|} \leq \frac{1}{2 \sqrt{\pi}} \frac{1}{\sqrt{\operatorname{Im} z}}, \quad z \in \mathbb{C}^{+} ;
$$

see, e.g., BW3, (2.6)].

Sometimes, a property of a de Branges space $\mathcal{H}=\mathcal{H}(E)$ will be defined in terms of the function $E$. Of course, proceeding in this way makes it necessary to show that the notion under consideration is well defined. For this purpose, the following facts are useful. Let $E_{1}, E_{2} \in \mathcal{H B}$ and assume that $\mathcal{H}\left(E_{1}\right)=\mathcal{H}\left(E_{2}\right)$. Then for some positive constants $c, C>0$, we have

$$
c \leq\left|\frac{E_{1}(z)}{E_{2}(z)}\right| \leq C, \quad z \in \mathbb{C}^{+} \cup \mathbb{R} .
$$

The function $E_{2}^{-1} E_{1}$ is of bounded type and has an analytic extension to some domain containing the closed half-plane $\mathbb{C}^{+} \cup \mathbb{R}$. This extension does not vanish at any point of $\mathbb{R} \cup \mathbb{C}^{+}$and satisfies $\operatorname{mt}\left(E_{2}^{-1} E_{1}\right)=0$. Thus, as is seen, e.g., from the theorem of Szego"-Solomentsev [RR, Theorem 3.13], it is outer.

It turns out that it is practical to use the notion of the mean type relative to a given de Branges space.

Definition 2.11. Let $\mathcal{H}$ be a de Branges space, let $D \subseteq \mathbb{C}$, and let $f: D \rightarrow \mathbb{C}$. Then we denote

$$
\operatorname{mt}_{\mathcal{H}} f:=\operatorname{mt} \frac{f}{\nabla_{\mathcal{H}}} .
$$

If $B$ is a set of functions, we again set $\operatorname{mt}_{\mathcal{H}} B:=\sup _{f \in B} \operatorname{mt}_{\mathcal{H}} f$.

Finally, we devote a couple of lines to the notion of the de Branges subspaces. 
Definition 2.12. Let $\mathcal{H}$ be a de Branges space. A closed subspace $\mathcal{L}$ of $\mathcal{H}$ is called a de Branges subspace of $\mathcal{H}$ if, endowed with the norm inherited from $\mathcal{H}$, it is itself a de Branges space.

A closed subspace $\mathcal{L}$ of $\mathcal{H}$ is a de Branges subspace of $\mathcal{H}$ if and only if

$$
F \in \mathcal{L} \Longrightarrow F^{\#} \in \mathcal{L} \text { and } F \in \mathcal{L}, w \in \mathbb{C}^{+}, F(w)=0 \Longrightarrow \frac{F(z)}{z-w} \in \mathcal{L} .
$$

Some de Branges subspaces are defined by an exponential growth restriction; namely, if $a \leq 0$, we denote

$$
\mathcal{H}_{(a)}:=\left\{F \in \mathcal{H}: \mathrm{mt}_{\mathcal{H}} F, \mathrm{mt}_{\mathcal{H}} F^{\#} \leq a\right\} .
$$

The fact that $\mathcal{H}_{(a)}$ is a de Branges subspace of $\mathcal{H}$ provided it contains a function that does not vanish identically was proved, e.g., in [KW $\$ 5]$. Moreover, the following is true:

(i) if $\mathcal{L}$ is a de Branges subspace of $\mathcal{H}$, then $\mathrm{mt}_{\mathcal{H}} \mathcal{L}=\mathrm{mt}_{\mathcal{H}} \nabla_{\mathcal{L}}$;

(ii) whenever $\mathcal{H}_{(a)} \neq\{0\}$, we have $\operatorname{mt}_{\mathcal{H}} \mathcal{H}_{(a)}=a$.

d. Monotone convergence theorem for nets of functions. We need the following version of the Lebesgue monotone convergence theorem, which deals with nondecreasing nets of nonnegative functions, rather than nondecreasing sequences. We shall thereby require an additional property of the functions under consideration, namely, lower semicontinuity. Other variants of the monotone convergence theorem put some restrictions on the order structure of the index set; see, e.g., [H-J].

Proposition 2.13. Let $X$ be a locally compact and $\sigma$-compact Hausdorff space, and let $\lambda$ be a positive Borel measure that is complete and regular and satisfies $\lambda(K)<\infty$ for all compact sets $K \subseteq X$.

Let $(I, \leq)$ be a directed set, and let $f_{i}: X \rightarrow[0, \infty], i \in I$, be a family of lower semicontinuous functions that is monotone nondecreasing, i.e., $f_{i}(x) \leq f_{j}(x), x \in X$, whenever $i \leq j$. Set $f(x):=\sup _{i \in I} f_{i}(x), x \in X$. Then

$$
\int_{X} f d \lambda=\sup _{i \in I} \int_{X} f_{i} d \lambda
$$

Proof. The inequality ' $\geq$ ' is trivial. For the proof of the reverse inequality, choose a function $g_{0}$ that is lower semicontinuous and everywhere positive and satisfies $\int_{X} g_{0} d \lambda=1$; e.g., we can take an appropriate step-function with open level sets, using $[\mathrm{R}$, Theorem 2.25]. Let $s$ be a nonnegative and bounded upper semicontinuous function with $s \leq f$. Moreover, let $\epsilon>0$ be given. By semicontinuity, the set

$$
E_{i}:=\left\{x \in X: f_{i}(x)-s(x)+\epsilon g_{0}(x)>0\right\}
$$

is open. Let $x \in X$ be given. Assume that $f(x)<\infty$, then there exists $i_{0} \in I$ such that $f_{i_{0}}(x)>f(x)-\epsilon g_{0}(x) \geq s(x)-\epsilon g_{0}(x)$, i.e., $x \in E_{i_{0}}$. If $f(x)=\infty$, then, since $s(x)<\infty$, again there exists $i_{0} \in I$ with $f_{i_{0}}(x)>s(x)-\epsilon g_{0}(x)$. We conclude that $X=\bigcup_{i \in I} E_{i}$. By $\sigma$-compactness there exists a countable subcover $\left\{E_{i_{1}}, E_{i_{2}}, \ldots\right\}$.

For each $n \in \mathbb{N}$, we choose an index $j_{n} \in I$ with $j_{n} \geq i_{1}, \ldots, i_{n}$. Since each $f_{i}$ is nonnegative and the family $\left(f_{i}\right)_{i \in I}$ is monotone, we obtain

$$
\sup _{i \in I} \int_{X} f_{i} d \lambda \geq \int_{X} f_{j_{n}} d \lambda \geq \int_{\bigcup_{k=1}^{n} E_{i_{k}}} f_{j_{n}} d \lambda \geq \int_{\bigcup_{k=1}^{n} E_{i_{k}}} s d \lambda-\epsilon \int_{\bigcup_{k=1}^{n} E_{i_{k}}} g_{0} d \lambda .
$$

Letting $n \in \mathbb{N}$ tend to infinity, we see that $\sup _{i \in I} \int_{X} f_{i} d \lambda \geq \int_{X} s d \lambda-\epsilon$. Since $\epsilon>0$ was arbitrary, this yields $\sup _{i \in I} \int_{X} f_{i} d \lambda \geq \int_{X} s d \lambda$. Finally, since the measure $\lambda$ is regular and $s$ is arbitrary, it follows that $\sup _{i \in I} \int_{X} f_{i} d \lambda \geq \int_{X} f d \lambda$, cf. [R, Theorem 2.25], which applies to each nonnegative step-function not exceeding $f$. 
Remark 2.14.

(i) Since $\left(f_{i}\right)_{i \in I}$ is monotone nondecreasing, the two suprema appearing in Proposition 2.13 are actually limits. Hence, by taking linear combinations, we obtain

$$
\int_{X} \lim _{i \in I} f_{i} d \lambda=\lim _{i \in I} \int_{X} f_{i} d \lambda
$$

whenever $\left(f_{i}\right)_{i \in I}$ satisfies the stated hypothesis and $\lambda$ is a complex (complete, regular) Borel measure.

(ii) Let us explicitly note that, in order to have (2.6), some assumption about the net $\left(f_{i}\right)_{i \in(I, \leq)}$ is needed. For example, let $I$ be the set of all finite subsets of $[0,1]$ ordered by set-theoretic inclusion. Moreover, let $f_{i}, i \in I$, be the indicator function of $i$. Then $f(x):=\sup _{i \in I} f_{i}(x)=1, x \in[0,1]$. Hence, integrating with respect to the Lebesgue measure, we get

$$
\int_{[0,1]} f d x=1 \quad \text { but } \int_{[0,1]} f_{i} d x=0, \quad i \in I .
$$

This example also explains why we cannot employ a compactness argument in Proposition 2.13. Since $f_{i}=0$ in $L^{1}(d x), i \in I$, we trivially have $\lim _{i \in I}\left(f_{i} d x\right)=0$ in $C([0,1])^{\prime}$. The point here is that, for a nondecreasing net of functions, unlike for a nondecreasing sequence, the $L^{1}$-limit and pointwise limit $\left(f_{i}\right)_{i \in I}$ need not coincide almost everywhere.

As a consequence of Proposition 2.13, we obtain the following two statements, and these are what will be needed later on in $\S 6$. A function $f$ is said to be superharmonic if $(-f)$ is subharmonic.

Corollary 2.15. Let $D \subseteq \mathbb{C}^{+}$be open, let $(I, \leq)$ be a directed set, and let $f_{i}: D \rightarrow$ $(-\infty, \infty], i \in I$, be a family of superharmonic functions. Assume that $\left(f_{i}\right)_{i \in I}$ is monotone nondecreasing and that, for some index $i_{0} \in I$, there exists a harmonic function $g: D \rightarrow$ $\mathbb{R}$ with $g \leq f_{i_{0}}$. Set $f:=\sup _{i \in I} f_{i}$. Then $f$ is superharmonic.

Proof. First, note that, being the supremum of a family of lower semicontinuous functions, $f$ is itself lower semicontinuous. Moreover, since the family $\left(f_{i}\right)_{i \in I}$ is nondecreasing, we have $f=\sup _{i \geq i_{0}} f_{i}$.

Let $U$ be a closed disk entirely contained in $D$, and let $a$ be its center and $r$ its radius. We have

$$
f_{i}(a) \geq \frac{1}{2 \pi} \int_{0}^{2 \pi} f_{i}\left(a+r e^{i t}\right) d t, \quad i \in I, \quad g(t)=\frac{1}{2 \pi} \int_{0}^{2 \pi} g\left(a+r e^{i t}\right) d t,
$$

and hence also

$$
\left(f_{i}-g\right)(a) \geq \frac{1}{2 \pi} \int_{0}^{2 \pi}\left(f_{i}-g\right)\left(a+r e^{i t}\right) d t, \quad i \in I .
$$

Taking the supremum over all $i \geq i_{0}$ yields

$$
f(a)-g(a) \geq \sup _{i \geq i_{0}} \frac{1}{2 \pi} \int_{0}^{2 \pi}\left(f_{i}-g\right)\left(a+r e^{i t}\right) d t .
$$

The family $\left(f_{i}-g\right)_{i \geq i_{0}}$ is a monotone nondecreasing family of nonnegative lower semicontinuous functions. Hence, by Proposition 2.13.

$$
\sup _{i \geq i_{0}} \frac{1}{2 \pi} \int_{0}^{2 \pi}\left(f_{i}-g\right)\left(a+r e^{i t}\right) d t=\frac{1}{2 \pi} \int_{0}^{2 \pi}(f-g)\left(a+r e^{i t}\right) d t .
$$


Using once again the mean value property of $g$, we conclude that

$$
f(a) \geq \frac{1}{2 \pi} \int_{0}^{2 \pi} f\left(a+r e^{i t}\right) d t .
$$

Corollary 2.16. Let $(I, \leq)$ be a directed set, and let $k_{i}: \mathbb{R} \rightarrow[0, \infty], i \in I$, be a monotone nondecreasing family of continuous functions. Assume that

$$
\log k_{i} \in L^{1}\left(\frac{d t}{1+t^{2}}\right), \quad i \in I, \quad \sup _{i \in I} \int_{\mathbb{R}} \log ^{+} k_{i}(t) \frac{d t}{1+t^{2}}<\infty .
$$

Denote $k(x):=\sup _{i \in I} k_{i}(x)=\lim _{i \in I} k_{i}(x), x \in \mathbb{R}$. Then $\log k \in L^{1}\left(\frac{d t}{1+t^{2}}\right)$ and

$$
\lim _{i \in I} \mathfrak{f}_{k_{i}}(z)=\mathfrak{f}_{k}(z), \quad z \in \mathbb{C}^{+} \text {. }
$$

Proof. Clearly, we have

$$
\log ^{-} k=-\min \{\log k, 0\} \leq \log ^{-} k_{i} \leq\left|\log k_{i}\right|, \quad i \in I .
$$

In particular, $\log ^{-} k \in L^{1}\left(\frac{d t}{1+t^{2}}\right)$.

The functions $\log ^{+} k_{i}, i \in I$, are continuous, nonnegative, and form a monotone nondecreasing family. Moreover, $\log ^{+} k=\sup _{i \in I} \log ^{+} k_{i}$. Thus, by Proposition 2.13 and the present assumption,

$$
\int_{\mathbb{R}} \log ^{+} k(t) \frac{d t}{1+t^{2}}=\int_{\mathbb{R}}\left[\sup _{i \in I} \log ^{+} k_{i}(t)\right] \frac{d t}{1+t^{2}}=\sup _{i \in I} \int_{\mathbb{R}} \log ^{+} k_{i}(t) \frac{d t}{1+t^{2}}<\infty .
$$

It follows that $\log k \in L^{1}\left(\frac{d t}{1+t^{2}}\right)$.

Let $z \in \mathbb{C}^{+}$be fixed. Then, as was mentioned in Remark 2.14 $(i)$, we may apply Proposition 2.13 with the complex measure

$$
d \lambda(t):=\left(\frac{1}{t-z}-\frac{t}{1+t^{2}}\right) d t .
$$

It follows that

$$
\lim _{i \in I} \int_{\mathbb{R}}\left(\frac{1}{t-z}-\frac{t}{1+t^{2}}\right) \log ^{+} k_{i}(t) d t=\lim _{i \in I} \int_{\mathbb{R}}\left(\frac{1}{t-z}-\frac{t}{1+t^{2}}\right) \log ^{+} k(t) d t .
$$

Fix $i_{0} \in I$. The functions $\log ^{-} k_{i_{0}}-\log ^{-} k_{i}, i \in I, i \geq i_{0}$, are continuous, nonnegative, and form a monotone nondecreasing family. Thus,

$$
\begin{aligned}
\lim _{i \in I} \int_{\mathbb{R}}( & \left.\frac{1}{t-z}-\frac{t}{1+t^{2}}\right) \log ^{-} k_{i}(t) d t \\
=- & \lim _{i \in I} \int_{\mathbb{R}}\left(\frac{1}{t-z}-\frac{t}{1+t^{2}}\right)\left[\log ^{-} k_{i_{0}}(t)-\log ^{-} k_{i}(t)\right] d t \\
& \quad+\int_{\mathbb{R}}\left(\frac{1}{t-z}-\frac{t}{1+t^{2}}\right) \log ^{-} k_{i_{0}}(t) d t=\int_{\mathbb{R}}\left(\frac{1}{t-z}-\frac{t}{1+t^{2}}\right) \log ^{-} k(t) d t .
\end{aligned}
$$

\section{§3. The FUnCTIONS $\mathfrak{m}_{B}$ AND $\mathfrak{m}_{B}^{\perp}$}

If $\mathcal{H}$ is a de Branges space, we denote its unit ball by $B(\mathcal{H})$, i.e.,

$$
B(\mathcal{H}):=\left\{F \in \mathcal{H}:\|F\|_{\mathcal{H}} \leq 1\right\} .
$$

Definition 3.1. Let $\mathcal{H}$ be a de Branges space, and let $B$ be a subset of its unit ball that contains a function not vanishing identically. We denote by $\mathfrak{m}_{B}$ the function

$$
\mathfrak{m}_{B}:\left\{\begin{aligned}
\mathbb{C}^{+} \cup \mathbb{R} & \rightarrow[0, \infty), \\
w & \mapsto \sup _{F \in B}|F(w)| .
\end{aligned}\right.
$$


Remark 3.2. The following facts should be noted explicitly.

(i) The supremum in the definition of $\mathfrak{m}_{B}$ is finite, because $B \subseteq B(\mathcal{H})$ implies that $|F(z)| \leq \nabla_{\mathcal{H}}(z), F \in B$.

(ii) We have $\mathfrak{m}_{B(\mathcal{H})}(w)=\nabla_{\mathcal{H}}(w), w \in \mathbb{C}^{+} \cup \mathbb{R}$.

(iii) If $B_{1} \subseteq B_{2}$, then $\mathfrak{m}_{B_{1}} \leq \mathfrak{m}_{B_{2}}$.

For particular sets $B$, namely, for unit balls $B_{\mathfrak{m}}(\mathcal{H})$ generated by majorization, we had already used this function in our previous work; cf. [BW4, §4]. There, for a majorant $\mathfrak{m}$ we defined the function

$$
\mathfrak{m}^{b}(w):=\sup _{F \in B_{\mathfrak{m}}(\mathcal{H})}|F(w)| \quad\left(=\mathfrak{m}_{B_{\mathfrak{m}}(\mathcal{H})}\right) .
$$

We keep this notation also in the present paper.

It will be important to know that the function $\mathfrak{m}_{B}$ is fairly smooth and reflects properties of $B$ in many respects. Below we collect some statements of this kind. As in BW4, Proposition 4.6], the proof is based on a normal family argument.

Lemma 3.3. Let $\mathcal{H}$ be a de Branges space, and let $B \subseteq B(\mathcal{H}), B \neq \varnothing,\{0\}$. Then:

(i) the function $\nabla_{\mathcal{H}}^{-1} \mathfrak{m}_{B}$ is continuous on $\mathbb{C}^{+} \cup \mathbb{R}$, and we have

$$
\lim _{z \rightarrow z_{0}, z \in \mathbb{C}^{+}} \frac{1}{\left|z-z_{0}\right|^{\mathfrak{d}_{B}\left(z_{0}\right)-\mathfrak{d}_{\mathcal{H}}\left(z_{0}\right)}} \frac{\mathfrak{m}_{B}(z)}{\nabla_{\mathcal{H}}(z)} \in(0, \infty), \quad z_{0} \in \mathbb{C}^{+} \cup \mathbb{R} ;
$$

(ii) $\mathfrak{d}_{\mathfrak{m}_{B}}=\mathfrak{d}_{B}$, and moreover, $\lim _{z \rightarrow w}\left|z-z_{0}\right|^{-\mathfrak{d}_{B}(w)} \mathfrak{m}_{B}(z) \in(0, \infty), w \in \mathbb{C}^{+} \cup \mathbb{R}$;

(iii) the function $\log \mathfrak{m}_{B}$ is subharmonic in $\mathbb{C}^{+}$;

(iv) $\mathrm{mt}_{\mathcal{H}} \mathfrak{m}_{B}=\mathrm{mt}_{\mathcal{H}} B$.

Proof. Let $E \in \mathcal{H} B$, and let $G$ be a domain that contains the closed half-plane $\mathbb{C}^{+} \cup \mathbb{R}$ and is such that $E$ has no nonreal zeros in $G$. Then the family $\mathcal{F}_{\mathcal{H}(E)}:=\left\{\left.\frac{F}{E}\right|_{G}: F \in B(\mathcal{H})\right\}$ is normal. Note that all elements of $\mathcal{F}_{\mathcal{H}(E)}$ are analytic, because $F \in \mathcal{H}(E)$ implies that $\left.\mathfrak{d}_{F}\right|_{\mathbb{R}} \geq\left.\mathfrak{d}_{E}\right|_{\mathbb{R}}$. If $E$ has no real zeros, this fact is clear. Otherwise, we can immediately reduce to this case.

Now we can prove $(i)$. Let $w \in \mathbb{C}^{+} \cup \mathbb{R}$, and set $n:=\mathfrak{d}_{B}(w)-\mathfrak{d}_{\mathcal{H}}(w) \geq 0$. Since $B \subseteq B(\mathcal{H})$, the family $\left\{E^{-1} F: F \in B\right\}$ is normal. Thus, also the family

$$
\mathcal{F}:=\left\{\frac{F(z)}{(z-w)^{n} E(z)}: F \in B\right\}
$$

is normal, and hence, equicontinuous. Using Lemma 2.10, we see that

$$
\frac{\mathfrak{m}_{B}(z)}{|z-w|^{n} \nabla_{\mathcal{H}}(z)}=\frac{|E(z)|}{\nabla_{\mathcal{H}}(z)} \cdot \frac{\mathfrak{m}_{B}(z)}{|z-w|^{n}|E(z)|}=\frac{|E(z)|}{\nabla_{\mathcal{H}}(z)} \cdot \sup _{F \in \mathcal{F}}\left|\frac{F(z)}{(z-w)^{n} E(z)}\right|
$$

is continuous. Moreover, since there exists a function $F \in B$ with $\mathfrak{d}_{F}(w)=\mathfrak{d}_{B}(w)$, the supremum on the right-hand side is positive. This shows $(i)$.

By Lemma 2.10 and property (i) in Subsection 2.9, assertion (ii) is an immediate consequence of (i).

We turn to the proof of (iii). By (i), the function $\log \mathfrak{m}_{B}$ is a continuous function from $\mathbb{C}^{+}$into $[-\infty, \infty)$. Moreover, it is the supremum of the subharmonic functions $\log |F(z)|$, $F \in B$. Thus, it is itself subharmonic.

Finally, we proceed to the proof of (iv). For each $F \in B$ we have $|F(z)| \leq \mathfrak{m}_{B}(z)$, $z \in \mathbb{C}^{+}$. Thus,

$$
\mathrm{mt}_{\mathcal{H}} B=\sup _{F \in B} \mathrm{mt}_{\mathcal{H}} F \leq \mathrm{mt}_{\mathcal{H}} \mathfrak{m}_{B} .
$$

Conversely, we have $B \subseteq \mathcal{H}_{(a)}$, where $a:=\operatorname{mt}_{\mathcal{H}} B$. It follows that $\mathfrak{m}_{B} \leq \nabla_{\mathcal{H}_{(a)}}$, whence

$$
\mathrm{mt}_{\mathcal{H}} \mathfrak{m}_{B} \leq \mathrm{mt}_{\mathcal{H}} \nabla_{\mathcal{H}_{(a)}}=a .
$$


Lemma 3.4. Let $\mathfrak{m}_{0} \in \operatorname{Adm} \mathcal{H}$, and denote the domain of $\mathfrak{m}_{0}$ by $D_{0} \subseteq \mathbb{C}^{+} \cup \mathbb{R}$. If $D \subseteq \mathbb{C}^{+} \cup \mathbb{R}$ is such that $D_{0} \subseteq \bar{D}$, and $a \in\left[\operatorname{mt}_{\mathcal{H}} B_{\mathfrak{m}_{0}}(\mathcal{H}), 0\right]$, then

$$
B_{\mathfrak{m}_{0}}(\mathcal{H})=B_{\left.\mathfrak{m}_{0}^{b}\right|_{D}}\left(\mathcal{H}_{(a)}\right) .
$$

Proof. If $F \in B_{\mathfrak{m}_{0}}(\mathcal{H})$, then $F \in \mathcal{H}_{(a)}$ and $|F(z)| \leq \mathfrak{m}_{0}^{b}(z), z \in \mathbb{C}^{+} \cup \mathbb{R}$. In particular, $F \in B_{\left.\mathfrak{m}_{0}^{b}\right|_{D}}\left(\mathcal{H}_{(a)}\right)$. Conversely, assume that $F$ belongs to this set. Then $F \in \mathcal{H}$ and $|F(z)| \leq \mathfrak{m}_{0}^{b}(z), z \in D$. By continuity, it follows that $|F(z)| \leq \mathfrak{m}_{0}^{b}(z), z \in \bar{D}$. Since $D_{0} \subseteq \bar{D}$, we obtain

$$
|F(z)| \leq \mathfrak{m}_{0}^{b}(z) \leq \mathfrak{m}_{0}(z), \quad z \in D_{0} .
$$

If $B$ is a subset of the unit ball of $\mathcal{H}$, define

$$
M_{\geq}(B):=\left\{\mathfrak{m} \in \operatorname{Adm} \mathcal{H}: B_{\mathfrak{m}}(\mathcal{H}) \supseteq B\right\},
$$

and set $B_{\geq}(B):=\beta\left(M_{\geq}(B)\right)$, where $\beta$ is the map $\mathfrak{m} \mapsto B_{\mathfrak{m}}(\mathcal{H})$; cf. (2.4).

Lemma 3.5. Let $\mathcal{H}$ be a de Branges space, and let $B \subseteq B(\mathcal{H}), B \neq \varnothing,\{0\}$.

(i) We have $\mathfrak{m} \in M_{\geq}(B)$ if and only if $\mathfrak{m} \in \operatorname{Adm} \mathcal{H}$ and $\mathfrak{m} \succcurlyeq \mathfrak{m}_{B}$.

(ii) Assume that for each $w \in \mathbb{C}^{+}$there exists an element $F \in B$ with $F(w) \neq 0$. Then $\mathfrak{m}_{B}$ belongs to $\operatorname{Adm} \mathcal{H}$ and is the smallest element of $M_{\geq}(B)$. The ball $B_{\mathfrak{m}_{B}}(\mathcal{H})$ is the smallest element of $B_{\geq}(B)$.

Proof. Let $D \subseteq \mathbb{C}^{+} \cup \mathbb{R}$ be the domain of $\mathfrak{m}$. Assume that $B_{\mathfrak{m}}(\mathcal{H}) \supseteq B$; then

$$
\mathfrak{m}_{B}(z)=\sup _{F \in B}|F(z)| \leq \sup _{F \in B_{\mathfrak{m}}(\mathcal{H})}|F(z)| \leq \mathfrak{m}(z), \quad z \in D .
$$

Conversely, if $\mathfrak{m} \geq\left.\mathfrak{m}_{B}\right|_{D}$ and $F \in B$, then

$$
|F(z)| \leq \mathfrak{m}_{B}(z) \leq \mathfrak{m}(z), \quad z \in D,
$$

whence $F \in B_{\mathfrak{m}}(\mathcal{H})$. This shows (i).

If there exists $F \in B$ with $F(w) \neq 0$, then $\mathfrak{m}_{B}(w)>0$. Hence, the assumption in (ii) together with the continuity of $\mathfrak{m}_{B}$ implies that $\mathfrak{d}_{\mathfrak{m}_{B}}(w)=0, w \in \mathbb{C}^{+}$. Since $B$ contains a function that does not vanish identically, we obtain $\mathfrak{m}_{B} \in \operatorname{Adm} \mathcal{H}$.

By (i), clearly, $\mathfrak{m}_{B}$ is the smallest element of $M_{\geq}(B)$. Since the map $\beta: \mathfrak{m} \mapsto B_{\mathfrak{m}}(\mathcal{H})$ preserves the respective orders and maps $M_{\geq}(B)$ surjectively onto $B_{\geq}(B)$, this implies that $\beta\left(\mathfrak{m}_{B}\right)$ is the smallest element of $B_{\geq}(B)$.

Lemma 3.6. Let $\mathcal{H}$ be a de Branges space, and let $B \subseteq B(\mathcal{H}), B \neq \varnothing,\{0\}$. Assume that $B$ is invariant with respect to division by Blaschke products; i.e., assume that for each function $F \in B$ and each Blaschke product $P$ for $\mathbb{C}^{+}$with $\left.\mathfrak{d}_{P}\right|_{\mathbb{C}^{+}} \leq\left.\mathfrak{d}_{F}\right|_{\mathbb{C}^{+}}$, we have $P^{-1} F \in B$. Then

$$
\mathfrak{m}_{B}(w)=\sup \left\{|F(w)|: F \in B, F \text { is zero-free in } \mathbb{C}^{+}\right\} .
$$

Proof. The inequality " $\geq$ " is fulfilled by the definition of $\mathfrak{m}_{B}$. Conversely, denote the supremum on the right-hand side by $M$, and let $F \in B$ be given. Let $P$ be the Blaschke product with $\left.\mathfrak{d}_{P}\right|_{\mathbb{C}^{+}}=\left.\mathfrak{d}_{F}\right|_{\mathbb{C}^{+}}$. Then $P^{-1} F \in B$, and hence

$$
|F(z)| \leq\left|\frac{F(z)}{P(z)}\right| \leq M, \quad z \in \mathbb{C}^{+} .
$$

The inequality " $\leq$ " follows.

With a subset $B$ of the unit ball $B(\mathcal{H})$, which is not too big in some sense, we can associate another function closely related to $B$. 
Definition 3.7. Let $\mathcal{H}$ be a de Branges space, and choose $E \in \mathcal{H} B$ with $\mathcal{H}=\mathcal{H}(E)$. Moreover, let $B \subseteq B(\mathcal{H}), B \neq \varnothing,\{0\}$, and assume that

$$
\int_{\mathbb{R}}\left(\log ^{+} \frac{\mathfrak{m}_{B}(t)}{|E(t)|}\right) \frac{d t}{1+t^{2}}<\infty .
$$

Then we define a function $\mathfrak{m}_{B}^{\perp}: \mathbb{C}^{+} \rightarrow[0, \infty)$ as

$$
\mathfrak{m}_{B}^{\perp}(z):=\left|e^{-i\left(\operatorname{mt}_{\mathcal{H}} B\right) \cdot z} \mathfrak{f}_{|E|^{-1} \mathfrak{m}_{B}}(z) E(z)\right|, \quad z \in \mathbb{C}^{+} .
$$

First, we need to show that $\mathfrak{m}_{B}^{\perp}$ is well defined.

Lemma 3.8. Let $B$ be a subset of $B(\mathcal{H}), B \neq \varnothing,\{0\}$, satisfying (3.1). Then the following statements hold true.

(i) We have $\int_{\mathbb{R}}\left|\log \left(|E|^{-1} \mathfrak{m}_{B}\right)\right|\left(1+t^{2}\right)^{-1} d t<\infty$. Hence the outer function $\mathfrak{f}_{|E|^{-1} \mathfrak{m}_{B}}$ in Definition 3.7 exists.

(ii) Neither the validity of (3.1) nor the function $\mathfrak{m}_{B}^{\perp}$ itself depends on the particular choice of $E$ in Definition 3.7.

Proof. Choose $F \in B \backslash\{0\}$. Then $E^{-1} F$ belongs to $H^{2}$ and does not vanish identically. Hence $\left(\log ^{-} x:=-\min \{\log x, 0\}\right)$,

$$
\int_{\mathbb{R}}\left(\log ^{-} \frac{\mathfrak{m}_{B}(t)}{|E(t)|}\right) \frac{d t}{1+t^{2}} \leq \int_{\mathbb{R}}\left(\log ^{-} \frac{|F(t)|}{|E(t)|}\right) \frac{d t}{1+t^{2}}<\infty .
$$

Together with (3.1), this gives $\log \left(|E|^{-1} \mathfrak{m}_{B}\right) \in L^{1}\left(\frac{d t}{1+t^{2}}\right)$.

The fact that the validity of (3.1) does not depend on the choice of $E$ follows from (2.5). Moreover, for any two functions $E_{1}, E_{2} \in \mathcal{H B}$ with $\mathcal{H}\left(E_{1}\right)=\mathcal{H}\left(E_{2}\right)$, we have $E_{1}^{-1} E_{2}=$ $\gamma \mathfrak{f}\left|E_{1}\right|^{-1}\left|E_{2}\right|$ with some $|\gamma|=1$. Consequently,

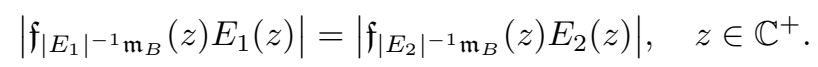

Now we show some properties of $\mathfrak{m}_{B}^{\perp}$ that will be needed later on.

Lemma 3.9. Let $B \subseteq B(\mathcal{H}), B \neq \varnothing,\{0\}$, and assume that (3.1) is fulfilled. Then:

(i) the function $\mathfrak{m}_{B}^{\perp}$ admits a continuous extension to the closed half-plane $\mathbb{C}^{+} \cup \mathbb{R}$, namely, by setting $\mathfrak{m}_{B}^{\perp}(x):=\mathfrak{m}_{B}(x), x \in \mathbb{R}$

(ii) we have $\mathfrak{m}_{B} \preccurlyeq \mathfrak{m}_{B}^{\perp}$.

Proof. In order to prove (i), we wish to apply Lemma 2.1 with the function $k(t):=$ $|E(t)|^{-1} \mathfrak{m}_{B}(t)$. The required hypotheses for this application were shown in Lemma $3.3(\mathrm{i})$. Actually, the limit relation (2.1) holds true for all $x_{0} \in \mathbb{R}$ when we set $n\left(x_{0}\right):=\mathfrak{d}_{B}\left(x_{0}\right)-$ $\mathfrak{d}_{E}\left(x_{0}\right)$. Remember here that, concerning continuity and zeros, it does not matter whether we consider the quotient $\nabla_{\mathcal{H}}^{-1} \mathfrak{m}_{B}$ or $|E|^{-1} \mathfrak{m}_{B}$; see Lemma 2.10(i).

If the function $F$ is chosen as in Lemma 2.1, we can write

$$
\mathfrak{m}_{B}^{\perp}(z)=\left|e^{-i\left(\operatorname{mt}_{\mathcal{H}} B\right) \cdot z}\right| \cdot|E(z) F(z)| \cdot\left|\frac{\mathfrak{f}|E|^{-1} \mathfrak{m}_{B}(z)}{F(z)}\right|, \quad z \in \mathbb{C}^{+} .
$$

From this formula, it is apparent that $\mathfrak{m}_{B}^{\perp}$ has a continuous extension $\tilde{\mathfrak{m}}_{B}^{\perp}$ to $\mathbb{C}^{+} \cup \mathbb{R}$. Moreover, for this extension we have

$$
\lim _{\substack{z \rightarrow x \\ z \in \mathbb{C}^{+} \cup \mathbb{R}}} \frac{\tilde{\mathfrak{m}}_{B}^{\perp}(z)}{|z-x|^{\mathfrak{d}_{B}(x)}} \in(0, \infty), \quad x \in \mathbb{R} .
$$

Again by Lemma 2.1 , the boundary values of $\mathfrak{m}_{B}^{\perp}$ along $\mathbb{R}$ are given by $\mathfrak{m}_{B}$. 
For the proof of (ii), let $F \in B$ be given. Then $E^{-1} F \in H^{2}$ and $\operatorname{mt}\left(E^{-1} F\right) \leq \mathrm{mt}_{\mathcal{H}} B$. Hence, the function

$$
g(z):=e^{i\left(\mathrm{mt}_{\mathcal{H}} B\right) \cdot z} \frac{F(z)}{E(z)}, \quad z \in \mathbb{C}^{+},
$$

belongs to the class $\mathcal{N}_{+}$. Actually, the function $g$ is continuous on $\mathbb{C}^{+} \cup \mathbb{R}$, and along the real axis we have

$$
|g(x)|=\left|\frac{F(x)}{E(x)}\right| \leq \frac{\mathfrak{m}_{B}(x)}{|E(x)|}, \quad x \in \mathbb{R}, \quad E(x) \neq 0 .
$$

By the Smirnov maximum principle, it follows that

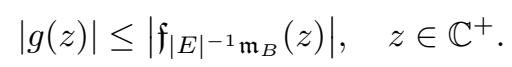

However, this merely says that $|F| \leq \mathfrak{m}_{B}^{\perp}$ throughout the half-plane $\mathbb{C}^{+}$. Thus,

$$
\mathfrak{m}_{B}(z)=\sup _{F \in B}|F(z)| \leq \mathfrak{m}_{B}^{\perp}(z), \quad z \in \mathbb{C}^{+} .
$$

\section{§4. Division By BlaschKe Products}

If majorization occurs along the real line, the situation is simple.

Lemma 4.1. Let $\mathcal{H}$ be a de Branges space, let $D \subseteq \mathbb{R}$, and let $\mathfrak{m} \in \operatorname{Adm}_{D} \mathcal{L}$. Moreover, let $\mathcal{L}$ be a de Branges subspace of $\mathcal{H}$. Then $B_{\mathfrak{m}}(\mathcal{H}) \cap \mathcal{L}\left(=B_{\mathfrak{m}}(\mathcal{L})\right)$ is invariant with respect to division by Blaschke products.

Proof. It is well known that each de Branges space is invariant with respect to division by Blaschke products. Since the norm in a de Branges space can be computed as a weighted $L^{2}$-integral along $\mathbb{R}$, even its unit ball has this property. For the same reason, majorization along any subset of $\mathbb{R}$ will be preserved under division by a Blaschke product.

Things change when majorization is required off the real axis.

Example 4.2. Consider the function

$$
E(z):=\left(1+\frac{z}{i}\right) \prod_{n \geq 2}\left(1+\frac{z}{i n^{2}}\right)^{2} .
$$

Then $E$ is of order $\frac{1}{2}$, belongs to $\mathcal{H} B$, and has no real zeros. By [B, Theorem 1], the space $\mathcal{H}:=\mathcal{H}(E)$ contains the set $\mathbb{C}[z]$ of all polynomials as a dense linear subspace. This implies that the chain of all de Branges subspaces $\mathcal{L}$ of $\mathcal{H}$ with $\mathfrak{d}_{\mathcal{L}}=0$ is equal to

$$
\left\{\mathbb{C}[z]_{n}: n \in \mathbb{N}_{0}\right\} \cup\{\mathcal{H}\} .
$$

Here $\mathbb{C}[z]_{n}$ denotes the set of all polynomials of degree at most $n$. Moreover, from $\underline{\mathrm{KW}}$, Theorem 3.4], it follows that every element of $\mathcal{H}(E)$ has order at most $\frac{1}{2}$.

Denote by $P_{0}$ the Blaschke product

$$
P_{0}(z):=\prod_{n \geq 2} \frac{1-z / i n^{2}}{1+z / i n^{2}} .
$$

Then the function

$$
F_{0}(z):=P_{0}(z) \frac{E(z)}{1+z / i}
$$

satisfies $F_{0}^{\#}=F_{0}$ and belongs to $\mathcal{H}$. The zeros of $F_{0}$ are all simple and located at the points $\pm i n^{2}, n \geq 2$. 
Let $c_{n}, n \geq 2$, be a sequence of positive real numbers with $c_{n} \rightarrow 0$. Choose radii $r_{n}$, $n \geq 2$, with $r_{n} \leq 1$, such that

$$
\left|F_{0}(z)\right| \leq c_{n}, \quad\left|z-i n^{2}\right| \leq r_{n}
$$

Let $D \subseteq \mathbb{C}^{+}$be any subset that contains the points $i n^{2}, n \geq 2$, and define $\mathfrak{m}: D \rightarrow[0, \infty)$ by

$$
\mathfrak{m}(z):= \begin{cases}c_{n} & \text { if }\left|z-i n^{2}\right| \leq r_{n}, \quad z \in D . \\ \left|F_{0}(z)\right| & \text { otherwise, }\end{cases}
$$

Then $\mathfrak{d}_{\mathfrak{m}}=0$ and $F_{0} \in R_{\mathfrak{m}}(\mathcal{H})$, whence $\mathfrak{m} \in \operatorname{Adm}_{D} \mathcal{H}$.

Assume that $F \in R_{\mathfrak{m}}(\mathcal{H})$ and that $F$ does not vanish in $\mathbb{C}^{+}$. Then, since $F$ has order $\leq \frac{1}{2}$, it has the property that $|F(i y)|$ is a monotone nondecreasing function of $y \geq 0$; see, e.g., $\mathrm{Bo}$. Since

$$
\liminf _{\substack{y \rightarrow+\infty \\ i y \in D}} \mathfrak{m}(i y) \leq \lim _{n \rightarrow+\infty} c_{n}=0,
$$

and $|F(i y)| \leq C \mathfrak{m}(i y)$, iy $\in D$, for some $C>0$, we also see that

$$
\liminf _{y \rightarrow+\infty}|F(i y)|=0 \text {. }
$$

Together with monotonicity, this implies that $F(i y)=0, y>0$, and we arrive at a contradiction.

It already follows that $R_{\mathfrak{m}}(\mathcal{H})$ cannot be invariant with respect to division by Blaschke products. We obtain even more information: since $R_{\mathfrak{m}}(\mathcal{H})$ is always invariant with respect to division by polynomials whose zeros lie off the real axis, every nonzero element of $R_{\mathfrak{m}}(\mathcal{H})$ has infinitely many zeros in $\mathbb{C}^{+}$. In particular, $R_{\mathfrak{m}}(\mathcal{H}) \cap \mathbb{C}[z]=\{0\}$, whence $\mathcal{R}_{\mathfrak{m}}(\mathcal{H})=\mathcal{H}$.

From this example we see that in general the situation can be complicated. Still, under an additional hypothesis on the majorant $\mathfrak{m}$, positive results can be obtained. The crucial notion in this context is the following.

Definition 4.3. Let $D \subseteq \mathbb{C}^{+}$be open, and let $\mathfrak{m}: D \rightarrow[0, \infty)$. We say that $\mathfrak{m}$ is $\log$-superharmonic if the function $(-\log \mathfrak{m})$ is subharmonic in $D$.

For log-superharmonic majorants, the Phragmén-Lindelöf principle, as stated in $\mathrm{RR}$, Theorem 6.2], can be applied. This gives the following theorem, which, in its turn, leads to results on dividing out zeros of elements of $R_{\mathfrak{m}}(\mathcal{H})$.

Theorem 4.4. Let $D$ be an open subset of $\mathbb{C}^{+}$, and let $\mathfrak{m} \in \operatorname{Adm}_{D} \mathcal{H}$ be log-superharmonic. Moreover, let $F \in B_{\mathfrak{m}}(\mathcal{H}) \backslash\{0\}$, and let $P$ be a Blaschke product for $\mathbb{C}^{+}$with $\left.\mathfrak{d}_{P}\right|_{\mathbb{C}^{+}} \leq\left.\mathfrak{d}_{F}\right|_{\mathbb{C}^{+}}$. Denote by $\alpha \in[0, \infty]$ the supremum of all nonnegative real numbers $\alpha^{\prime}$ such that

$$
\alpha^{\prime}\left|\frac{F(\zeta)}{P(\zeta)}\right| \leq \liminf _{\substack{z \rightarrow \zeta \\ z \in D}} \mathfrak{m}(z), \quad \zeta \in \partial D \backslash \mathbb{R}
$$

Then

We always have

$$
\left|\frac{F(z)}{P(z)}\right| \leq \max \left\{\alpha^{-1},\|F\|_{\mathfrak{m}}\right\} \mathfrak{m}(z), \quad z \in D
$$

$$
\alpha \geq \inf _{\zeta \in \partial D \backslash \mathbb{R}}|P(\zeta)|
$$


Proof. Step 1. Let $F \in B_{\mathfrak{m}}(\mathcal{H})$, let $P$ be a finite Blaschke product with $\left.\mathfrak{d}_{P}\right|_{\mathbb{C}^{+}} \leq\left.\mathfrak{d}_{F}\right|_{\mathbb{C}^{+}}$, and let $\alpha^{\prime} \in(0,1]$ be such that (4.1) is fulfilled.

Consider the function

$$
u(z)=-\log \mathfrak{m}(z)+\log \left|\alpha^{\prime} \frac{F(z)}{P(z)}\right|, \quad z \in D,
$$

where $u(z)$ is interpreted as $-\infty$ if $\mathfrak{m}(z)=\infty$ or $P(z)^{-1} F(z)=0$. Note that always $\mathfrak{m}(z)>0$, because $z \in D \subseteq \mathbb{C}^{+}$. Then $u$ is subharmonic in $D$. Observe that if $P(z) \neq 0$ for some $z \in D$, then we can also write

$$
u(z)=-\log \mathfrak{m}(z)+\log |F(z)|+\log \alpha^{\prime}-\log |P(z)| .
$$

We represent the boundary $\partial_{\infty} D$ of $D$ in the extended complex plane $\mathbb{C} \cup\{\infty\}$ as the disjoint union of the sets

$$
R:=\partial D \backslash \mathbb{R}, \quad S:=(\partial D \cap \mathbb{R}) \cup\{\infty\} .
$$

By our hypothesis (4.1), for $\zeta \in R$,

$$
\lim _{\substack{z \rightarrow \zeta \\ z \in D}} \alpha^{\prime}\left|\frac{F(z)}{P(z)}\right|=\alpha^{\prime}\left|\frac{F(\zeta)}{P(\zeta)}\right| \leq \liminf _{\substack{z \rightarrow \zeta \\ z \in D}} \mathfrak{m}(z),
$$

and therefore,

$$
\limsup _{\substack{z \rightarrow \zeta \\ z \in D}}(-\log \mathfrak{m}(z)) \leq-\lim _{\substack{z \rightarrow \zeta \\ z \in D}} \log \left(\alpha^{\prime}\left|\frac{F(z)}{P(z)}\right|\right) .
$$

Since $\mathfrak{d}_{\mathfrak{m}}(\zeta)=0$ and $P^{-1} F$ is analytic at $\zeta$, neither of these limits can be equal to $+\infty$. It follows that

$$
\limsup _{\substack{z \rightarrow \zeta \\ z \in D}} u(z)=\limsup _{\substack{z \rightarrow \zeta \\ z \in D}}(-\log \mathfrak{m}(z))+\lim _{\substack{z \rightarrow \zeta \\ z \in D}} \log \left(\alpha^{\prime}\left|\frac{F(z)}{P(z)}\right|\right) \leq 0 .
$$

Next, let $\zeta \in S, \zeta \neq \infty$, and let $\epsilon>0$ be given. Since $\zeta \in \mathbb{R}$, there exists a neighborhood $U$ of $\zeta$ such that

$$
-\log |P(z)|<\epsilon, \quad z \in U .
$$

Since $F \in B_{\mathfrak{m}}(\mathcal{H})$ and $\alpha^{\prime} \leq 1$, we have

$$
-\log \mathfrak{m}(z)+\log |F(z)|+\log \alpha^{\prime} \leq 0, \quad z \in U \cap D .
$$

Thus, by (4.2), $u(z)<\epsilon$ for $z \in U \cap D$.

Finally, consider $\zeta=\infty$, and let $\epsilon>0$ be given. Since $P$ is a finite Blaschke product, there exists a neighborhood $U$ of $\zeta$ such that $-\log |P(z)|<\epsilon, z \in U \backslash\{\infty\}$. In the same way as above, now we obtain $u(z)<\epsilon, z \in U \cap D$.

We can apply the Phragmén-Lindelöf principle, cf. [RR, Theorem 6.2], to conclude that $u(z) \leq 0$ throughout $D$, i.e.,

$$
\alpha^{\prime}\left|\frac{F(z)}{P(z)}\right| \leq \mathfrak{m}(z), \quad z \in D .
$$

Since $\alpha^{\prime} \leq 1$ and $\|F\|_{\mathcal{H}} \leq 1$, we also have $\left\|\alpha^{\prime} P^{-1} F\right\|_{\mathcal{H}} \leq 1$. Thus, $\alpha^{\prime} P^{-1} F \in B_{\mathfrak{m}}(\mathcal{H})$.

Step 2. Let $F \in B_{\mathfrak{m}}(\mathcal{H})$, let $P$ be an arbitrary Blaschke product with $\left.\mathfrak{d}_{P}\right|_{\mathbb{C}^{+}} \leq\left.\mathfrak{d}_{F}\right|_{\mathbb{C}^{+}}$, and let $\alpha^{\prime} \in(0,1]$ be such that (4.1) is fulfilled.

For $N \in \mathbb{N}$, denote by $P_{N}$ the $N$ th partial product of $P$. Since $\left|P_{N}(z)\right| \geq|P(z)|$, $z \in \mathbb{C}^{+}$, the number $\alpha^{\prime}$ also satisfies (4.1) with $P_{N}$ in place of $P$. Therefore, by Step 1 , $\alpha^{\prime} P_{N}^{-1} F \in B_{\mathfrak{m}}(\mathcal{H})$. Since $B_{\mathfrak{m}}(\mathcal{H})$ is weakly compact, there is a sequence $\left(N_{k}\right)_{k \in \mathbb{N}}$ and a function $G \in B_{\mathfrak{m}}(\mathcal{H})$ such that $\lim _{k \rightarrow \infty}^{w} \alpha^{\prime} P_{N}^{-1} F=G$. Since weak convergence implies pointwise convergence, it follows that $G=\alpha^{\prime} P^{-1} F$. 
Step 3. Set

$$
\begin{aligned}
\alpha & :=\sup \left\{\alpha^{\prime} \geq 0: \text { (4.1) holds }\right\} \\
\beta & :=\max \left\{\beta^{\prime} \geq 0: \beta^{\prime} \frac{F}{P} \in B_{\mathfrak{m}}(\mathcal{H})\right\} .
\end{aligned}
$$

Then

$$
1 \geq\left\|\beta \frac{F}{P}\right\|_{\mathfrak{m}} \geq \beta\|F\|_{\mathfrak{m}},
$$

i.e., $\beta \leq\|F\|_{\mathfrak{m}}^{-1}$. Also, $\beta\left|P(z)^{-1} F(z)\right| \leq \mathfrak{m}(z), z \in D$, so that (4.1) holds true with $\alpha^{\prime}:=\beta$, i.e., $\beta \leq \alpha$.

On the other hand, let $0<\beta^{\prime}<\min \left\{\alpha,\|F\|_{\mathfrak{m}}^{-1}\right\}$. Then $G:=\|F\|_{\mathfrak{m}}^{-1} F \in B_{\mathfrak{m}}(\mathcal{H})$ and $\alpha^{\prime}:=\beta^{\prime}\|F\|_{\mathfrak{m}}$ satisfies (4.1) for $G$. Moreover, $\alpha^{\prime}<1$, whence

$$
\beta^{\prime} \frac{F}{P}=\alpha^{\prime} \frac{G}{P} \in B_{\mathfrak{m}}(\mathcal{H}) .
$$

This shows that $\beta^{\prime} \leq \beta$. We conclude that $\beta=\min \left\{\alpha,\|F\|_{\mathfrak{m}}^{-1}\right\}$.

Step 4. The estimate $\alpha \geq \inf _{\zeta \in \partial D}|P(\zeta)|$ follows, because $F \in B_{\mathfrak{m}}(\mathcal{H})$ implies that $|F(\zeta)| \leq \liminf _{\substack{z \rightarrow \zeta \\ z \in D}} \mathfrak{m}(z), \zeta \in \partial D$.

Now we demonstrate in two particular situations how Theorem 4.4 can be applied.

Corollary 4.5. Let $\mathfrak{m} \in \operatorname{Adm}_{\mathbb{C}^{+}} \mathcal{H}$ be $\log$-superharmonic. If $F \in B_{\mathfrak{m}}(\mathcal{H})$ and $P$ is a Blaschke product for $\mathbb{C}^{+}$with $\left.\mathfrak{d}_{P}\right|_{\mathbb{C}^{+}} \leq\left.\mathfrak{d}_{F}\right|_{\mathbb{C}^{+}}$, then $P^{-1} F \in B_{\mathfrak{m}}(\mathcal{H})$.

Proof. This follows from Theorem 4.4, because $\partial \mathbb{C}^{+}=\mathbb{R}$ and thus (4.1) is trivially satisfied with $\alpha^{\prime}=1$.

Combining this statement with Example 4.2 leads to the following observation.

Remark 4.6. A log-superharmonic majorant $\mathfrak{m} \in \operatorname{Adm}_{\mathbb{C}^{+}} \mathcal{H}$ that generates the same unit ball does not exist for every majorant $\mathfrak{m}_{0}$.

Note that this contrasts the situation that prevails for log-subharmonic majorants, i.e., the majorants $\mathfrak{m} \in \operatorname{Adm}_{\mathbb{C}^{+}} \mathcal{H}$ for which the function $\log \mathfrak{m}$ is subharmonic. Remember that, for each majorant $\mathfrak{m}_{0} \in \operatorname{Adm} \mathcal{H}$, the function $\left.\mathfrak{m}_{0}^{b}\right|_{\mathbb{C}^{+}}$is $\log$-subharmonic and $B_{\mathfrak{m}_{0}}(\mathcal{H})=B_{\left.\mathfrak{m}_{0}^{b}\right|_{\mathbb{C}^{+}}}(\mathcal{H})$; cf. Lemma 3.3 and Lemma 3.4

Corollary 4.7. Let $\delta \in\left(0, \frac{\pi}{2}\right)$, let $D$ be the Stolz angle $D:=\{z \in \mathbb{C}: \delta<\arg z<$ $\pi-\delta\}$, and let $\mathfrak{m} \in \operatorname{Adm}_{D} \mathcal{H}$. If $F \in B_{\mathfrak{m}}(\mathcal{H})$ and $P$ is a Blaschke product for $\mathbb{C}^{+}$with $\left.\mathfrak{d}_{P}\right|_{\mathbb{C}^{+}} \leq\left.\mathfrak{d}_{F}\right|_{\mathbb{C}^{+}}$and

$$
\sum_{w \in \mathbb{C}^{+}} \mathfrak{d}_{P}(w) \frac{\operatorname{Im} w}{|w|}<\infty
$$

then $P^{-1} F \in R_{\mathfrak{m}}(\mathcal{H})$.

Proof. We write

$$
P(z)=\prod_{k=1}^{\infty} \frac{1-z / z_{k}}{1-z / \overline{z_{k}}}
$$

with the zeros repeated according to their multiplicities. We have

$$
2 \log |P(z)|=\sum_{w \in \mathbb{C}^{+}} \mathfrak{d}_{P}(w) \log \left(1-\frac{4 \operatorname{Im} z \operatorname{Im} w}{|z-\bar{w}|^{2}}\right)<\infty .
$$


For each $\delta \in\left(0, \frac{\pi}{2}\right)$, there is a constant $C=C(\delta)>0$ such that $|z-\bar{w}| \geq C(|z|+|w|)$ for any $z \in D, w \in \mathbb{C}^{+}$. Now from (4.3) and (4.4) it follows that there exists $N=N(\delta) \in \mathbb{N}$ such that

$$
P_{N}(z):=\prod_{k=N}^{\infty} \frac{1-z / z_{k}}{1-z / \overline{z_{k}}}
$$

satisfies $\left|P_{N}(z)\right| \geq 1 / 2$ for any $z \in D$. Hence, $\left(P_{N}\right)^{-1} F \in R_{\mathfrak{m}}(\mathcal{H})$.

\section{§5. UNIT BALLS OF LOG-SUPERHARMONIC MAJORANTS}

Our aim in the present section is to investigate what subsets $B$ of a de Branges space $\mathcal{H}$ can be realized as $B_{\mathfrak{m}}(\mathcal{H})$ with some $\log$-superharmonic majorant $\mathfrak{m} \in \operatorname{Adm}_{\mathbb{C}^{+}} \mathcal{H}$. It is an interesting result that such balls can also be realized by majorization along $\mathbb{R}$ in conjunction with a restriction of exponential growth towards infinity; cf. Theorem 5.3. We shall also discuss representability with more particular kinds of majorants, which appear naturally in this context, namely, the $\mathcal{N}_{+}$-majorants and $H^{p}$-majorants; cf. Definitions 5.1 and 5.13 .

a. Representability by log-superharmonic majorants. In the study of log-superharmonic majorants defined on all of $\mathbb{C}^{+}$, a smaller class of majorants plays a key role.

Definition 5.1. Let $\mathcal{H}$ be a de Branges space and choose $E \in \mathcal{H} B$ with $\mathcal{H}=\mathcal{H}(E)$. A function $\mathfrak{m} \in \operatorname{Adm}_{\mathbb{C}^{+}} \mathcal{H}$ is called an $\mathcal{N}_{+}$-majorant for $\mathcal{H}$ if it is of the form

$$
\mathfrak{m}(z)=\left|e^{-i a z} f(z) E(z)\right|, \quad z \in \mathbb{C}^{+},
$$

with some $a \leq 0$ and $f$ being outer for $\mathcal{N}$.

The fact whether or not a given function $\mathfrak{m}$ is an $\mathcal{N}_{+}$-majorant for $\mathcal{H}$ does not depend on the particular choice of the function $E$ in Definition 5.1. Indeed, for any two functions $E_{1}, E_{2} \in \mathcal{H B}$ with $\mathcal{H}\left(E_{1}\right)=\mathcal{H}\left(E_{2}\right)$, the quotient $E_{1}^{-1} E_{2}$ is outer.

Example 5.2. Let $\mathcal{H}=\mathcal{H}(E)$ be a de Branges space, and let $B \subseteq B(\mathcal{H}), B \neq \varnothing,\{0\}$. Assume that (3.1) is fulfilled, i.e., $\int_{\mathbb{R}}\left[\log ^{+}\left(|E|^{-1} \mathfrak{m}_{B}\right)\right]\left(1+t^{2}\right)^{-1} d t<\infty$. Then the function $\mathfrak{m}_{B}^{\perp}$ is an $\mathcal{N}_{+}$-majorant. We have

$$
B \subseteq B_{\mathfrak{m}_{B}^{\perp}}(\mathcal{H}) .
$$

These assertions are immediate from the respective definitions and the fact that $\mathfrak{m}_{B} \frac{\downarrow}{\succcurlyeq}$ $\mathfrak{m}_{B} ;$ cf. Lemma 3.9

For each $\mathcal{N}_{+}$-majorant $\mathfrak{m}$, the function $\log \mathfrak{m}$ is harmonic in $\mathbb{C}^{+}$. Hence, in general, the set of all $\mathcal{N}_{+}$-majorants is a fairly small subset of the set of all log-superharmonic majorants.

Theorem 5.3. Let $\mathcal{H}$ be a de Branges space, and let $E \in \mathcal{H B}$ be such that $\mathcal{H}=\mathcal{H}(E)$. Moreover, let $B \subseteq B(\mathcal{H}), B \neq \varnothing,\{0\}$. The following statements are equivalent.

(i) There exists a $\log$-superharmonic majorant $\mathfrak{m} \in \operatorname{Adm}_{\mathbb{C}^{+}} \mathcal{H}$ such that $B=B_{\mathfrak{m}}(\mathcal{H})$.

(ii) There exists an $\mathcal{N}_{+}$-majorant $\mathfrak{m} \in \operatorname{Adm}_{\mathbb{C}^{+}} \mathcal{H}$ such that $B=B_{\mathfrak{m}}(\mathcal{H})$.

(iii) There exists a subset $D \subseteq \mathbb{R}$ such that $\mathbb{R} \backslash D$ has measure zero, a number $a \leq 0$, and a measurable majorant $\mathfrak{m} \in \operatorname{Adm}_{D} \mathcal{H}_{(a)}$ satisfying

$$
\int_{D}\left(\log ^{+} \frac{\mathfrak{m}(t)}{|E(t)|}\right) \frac{d t}{1+t^{2}}<\infty
$$

such that $B=B_{\mathfrak{m}}\left(\mathcal{H}_{(a)}\right)$. 
(iv) We have $B=B_{\left.\mathfrak{m}_{B}\right|_{\mathbb{R}}}\left(\mathcal{H}_{\left(\mathrm{mt}_{\mathcal{H}} B\right)}\right)$ and

$$
\int_{D}\left(\log ^{+} \frac{\mathfrak{m}_{B}(t)}{|E(t)|}\right) \frac{d t}{1+t^{2}}<\infty .
$$

Proof. First, we note that the implications (ii) $\Longrightarrow$ (i) and (iv) $\Longrightarrow$ (iii) are trivial. We are going to prove the implications

$$
\text { (i) } \Longrightarrow \text { (ii) } \Longrightarrow \text { (iv), (iii) } \Longrightarrow \text { (ii). }
$$

Step 1. Assume that $\mathfrak{m} \in \operatorname{Adm}_{\mathbb{C}^{+}} \mathcal{H}$ is $\log$-superharmonic. At this step we show that then the subharmonic function $\log \left(|E(z)|^{-1} \mathfrak{m}^{\mathrm{b}}(z)\right), z \in \mathbb{C}^{+}$, has a nonnegative harmonic majorant.

Denote $\mathcal{F}:=\left\{F \in B_{\mathfrak{m}}(\mathcal{H}): F\right.$ is zero-free in $\left.\mathbb{C}^{+}\right\}$. For any $F \in \mathcal{F}$, we have

$$
\log \left|\frac{F}{E}\right| \leq \log \frac{\mathfrak{m}^{b}}{|E|} \leq \log \frac{\mathfrak{m}}{|E|}
$$

throughout the half-plane $\mathbb{C}^{+}$. Since, by Corollary $4.5, \mathcal{F}$ is nonempty, this tells us, in particular, that the superharmonic function $\log \left(|E|^{-1} \mathfrak{m}\right)$ has a harmonic minorant. Thus, it has the largest harmonic minorant, which we denote by $h$. For each element $F \in \mathcal{F}$, the function $\log \left(|E|^{-1}|F|\right)$ is a harmonic minorant of $\log \left(|E|^{-1} \mathfrak{m}\right)$, and hence does not exceed $h$. Lemma 3.6 implies that also $\log \left(|E|^{-1} \mathfrak{m}^{b}\right) \leq h$. Thus, the subharmonic function $\log \left(|E|^{-1} \mathfrak{m}^{b}\right)$ has a harmonic majorant. Therefore, it has the smallest harmonic majorant, which we denote by $h^{b}$. Clearly, $h^{b} \leq h$, and altogether

$$
\log \left|\frac{F}{E}\right| \leq \log \frac{\mathfrak{m}^{b}}{|E|} \leq h^{b} \leq h \leq \log \frac{\mathfrak{m}}{|E|}, \quad F \in \mathcal{F} .
$$

Fix $F_{0} \in \mathcal{F}$. Since $E^{-1} F_{0} \in H^{2} \subseteq \mathcal{N}$, the subharmonic function $\log ^{+}\left|E^{-1} F_{0}\right|$ has a harmonic majorant; we denote one such function by $k$. Define

$$
k^{b}:=h^{b}+k-\log \left|\frac{F_{0}}{E}\right| .
$$

Then $k^{b}$ is harmonic and

$$
k^{b}=h^{b}+\underbrace{\left(k-\log \left|\frac{F_{0}}{E}\right|\right)}_{\geq 0} \geq \log \frac{\mathfrak{m}^{b}}{|E|}, \quad k^{b}=\underbrace{\left(h^{b}-\log \left|\frac{F_{0}}{E}\right|\right)}_{\geq 0}+k \geq 0 .
$$

We see that $k^{b}$ is a nonnegative harmonic majorant of $\log \left(|E|^{-1} \mathfrak{m}^{b}\right)$.

Step 2. Assume that $\mathfrak{m} \in \operatorname{Adm}_{\mathbb{C}^{+}} \mathcal{H}$ is log-superharmonic. At this step we determine the least harmonic majorant $h^{b}$ of $\log \left(|E|^{-1} \mathfrak{m}^{b}\right)$ explicitly.

At Step 1 we showed that $\log \left(|E|^{-1} \mathfrak{m}^{b}\right)$ has a nonnegative harmonic majorant. Hence, the least harmonic majorant $h^{\mathrm{b}}$ of this function can be represented as a Poisson integral:

$$
h^{b}(z)=a y+\frac{y}{\pi} \int_{\mathbb{R}} \frac{d \mu(t)}{(t-x)^{2}+y^{2}}, \quad z=x+i y \in \mathbb{C}^{+},
$$

where $a \in \mathbb{R}$, and $\mu$ is a real (signed) Borel measure on $\mathbb{R}$ satisfying $\int_{\mathbb{R}}\left(1+t^{2}\right)^{-1} d|\mu|(t)<\infty$. We employ the Szegö-Solomentsev theorem to determine the data $a$ and $\mu$; see, e.g., $\underline{R}$, Theorem 3.13], where the version for the unit disk is stated.

Since $|E|^{-1} \mathfrak{m}^{b}$ has a continuous extension to the closed half-plane $\mathbb{C}^{+} \cup \mathbb{R}$, the RadonNikodym derivative of $\mu$ with respect to the Lebesgue measure is equal to $\log \left(|E|^{-1} \mathfrak{m}^{b}\right)$; see [RR, Theorem 3.13(iv)]. Since $|E|^{-1} \mathfrak{m}^{b}$ does not vanish on $\mathbb{R}$ with the possible exception of a discrete set where it tends to zero polynomially, the measure $\mu$ is absolutely 
continuous with respect to the Lebesgue measure. To see this, use [RR, Theorem 3.13(ii)], e.g., with the function $\varphi(t):=t^{2}$.

Set $B:=B_{\mathfrak{m}}(\mathcal{H})$. We showed that $d \mu=\log \left(|E|^{-1} \mathfrak{m}^{b}\right) d t$; in particular, the integral $\int_{\mathbb{R}}\left|\log \left(|E|^{-1} \mathfrak{m}^{b}\right)\right|\left(1+t^{2}\right)^{-1} d t$ converges. Hence, the function $\mathfrak{m}_{B}^{\perp}$ is well defined. Moreover, $\log \left(|E|^{-1} \mathfrak{m}_{B}^{\perp}\right)$ is harmonic and is greater than or equal to $\log \left(|E|^{-1} \mathfrak{m}^{b}\right)$; cf. Lemma 3.9.(ii). Since $h^{b}$ is the least harmonic majorant of $\log \left(|E|^{-1} \mathfrak{m}^{b}\right)$, this implies that

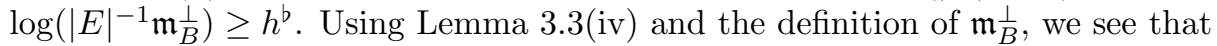

$$
\mathrm{mt}_{\mathcal{H}} B=\mathrm{mt} \frac{\mathfrak{m}^{b}}{|E|} \leq \underbrace{\operatorname{mt}\left[\exp \left(h^{b}\right)\right]}_{=a} \leq \mathrm{mt} \frac{\mathfrak{m}_{B}^{\perp}}{|E|}=\mathrm{mt}_{\mathcal{H}} B,
$$

i.e., $a=\mathrm{mt}_{\mathcal{H}} B$. Altogether, we obtain

$$
h^{b}=\log \frac{\mathfrak{m}_{B}^{\perp}}{|E|} .
$$

Step 3. (i) $\Longrightarrow$ (ii). Now it is easy to establish this implication. Let $B$ be given, and assume that $\mathfrak{m} \in \operatorname{Adm}_{\mathbb{C}^{+}} \mathcal{H}$ is $\log$-superharmonic and satisfies $B=B_{\mathfrak{m}}(\mathcal{H})$. By (5.2) and (5.3), we have

$$
\mathfrak{m}^{b} \leq|E| \cdot \exp \left(h^{b}\right)=\mathfrak{m}_{B}^{\perp} \leq \mathfrak{m},
$$

and it follows that $B_{\mathfrak{m}}(\mathcal{H})=B_{\mathfrak{m}^{\perp}}(\mathcal{H})$.

Step 4. Let $\mathfrak{m}: \mathbb{C}^{+} \rightarrow[0, \infty)$ be a function of the form

$$
\mathfrak{m}(z)=\left|e^{-i a z} f(z) E(z)\right|, \quad z \in \mathbb{C}^{+},
$$

where $f$ is outer, and $a=\operatorname{mt}_{\mathcal{H}} \mathfrak{m} \leq 0$. Denote by $D \subseteq \mathbb{R}$ the set of all points $x \in \mathbb{R}$ such that the nontangential limit $f^{*}(x):=\lim _{z \rightarrow x} f(z)$ exists and $E(x) \neq 0$, and set

$$
\mathfrak{m}^{*}(x):=\lim _{z \rightarrow x} \mathfrak{m}(z)=\left|f^{*}(x) E(x)\right|, \quad x \in D .
$$

Our aim at this step is to show that

$$
B_{\mathfrak{m}}(\mathcal{H})=B_{\mathfrak{m}^{b} \mid \mathbb{R}}\left(\mathcal{H}_{(b)}\right)=B_{\mathfrak{m}^{*}}\left(\mathcal{H}_{(b)}\right), \quad b \in\left[\operatorname{mt}_{\mathcal{H}} B_{\mathfrak{m}}(\mathcal{H}), \mathrm{mt}_{\mathcal{H}} \mathfrak{m}\right] .
$$

Thereby, the inclusion " $\subseteq$ " in the first asserted equality is true because $b \geq \operatorname{mt}_{\mathcal{H}} B_{\mathfrak{m}}(\mathcal{H})$. The inclusion " $\subseteq$ " in the second equality follows from

$$
\mathfrak{m}^{b}(x)=\lim _{z \widehat{\rightarrow} x} \mathfrak{m}^{b}(z) \leq \lim _{z \widehat{\rightarrow} x} \mathfrak{m}(z)=\mathfrak{m}^{*}(x), \quad x \in D,
$$

i.e., $\left.\mathfrak{m}^{b}\right|_{\mathbb{R}} \preccurlyeq \mathfrak{m}^{*}$. Let $F \in B_{\mathfrak{m}^{*}}\left(\mathcal{H}_{(b)}\right)$ be given and consider the function

$$
g(z):=e^{i a z} \frac{F(z)}{E(z)}, \quad z \in \mathbb{C}^{+} \cup \mathbb{R} .
$$

Note that $g$ is continuous on $\mathbb{C}^{+} \cup \mathbb{R}$. Since $F \in \mathcal{H}_{(b)} \subseteq \mathcal{H}$, we have $E^{-1} F \in H^{2}$ and $\operatorname{mt}\left(E^{-1} F\right) \leq b \leq a$. It follows that $g \in \mathcal{N}_{+}$. Along the real axis we have

$$
|g(x)| \leq \frac{\mathfrak{m}^{*}(x)}{|E(x)|}=\left|f^{*}(x)\right|, \quad x \in D .
$$

Since $f$ is outer, the Smirnov maximum principle implies that the inequality $|g(z)| \leq$ $|f(z)|$ prevails throughout the half-plane $\mathbb{C}^{+}$. Thus,

$$
|F(z)| \leq\left|e^{-i a z} E(z)\right| \cdot|f(z)|=\mathfrak{m}(z), \quad z \in \mathbb{C}^{+},
$$

and we obtain $F \in B_{\mathfrak{m}}(\mathcal{H})$. The proof of (5.6) is complete. 
Step 5. (ii) $\Longrightarrow$ (iv). Again, now this implication is easy to check. Let $B$ be given, and assume that $\mathfrak{m} \in \operatorname{Adm}_{\mathbb{C}^{+}} \mathcal{H}$ is an $\mathcal{N}_{+}$-majorant with $B=B_{\mathfrak{m}}(\mathcal{H})$. What we showed at Step 4 applies to $\mathfrak{m}$. The first identity in (5.6) with $b=\operatorname{mt}_{\mathcal{H}} B_{\mathfrak{m}}(\mathcal{H})$, however, is simply the first condition in (iv). Moreover, from (5.7) and the fact that $\mathbb{R} \backslash D$ has measure zero, we see that

$$
\int_{\mathbb{R}}\left(\log ^{+} \frac{\mathfrak{m}^{b}(t)}{|E(t)|}\right) \frac{d t}{1+t^{2}} \leq \int_{D}\left(\log ^{+} \frac{\mathfrak{m}^{*}(t)}{|E(t)|}\right) \frac{d t}{1+t^{2}}=\int_{D}\left[\log ^{+} f^{*}(t)\right] \frac{d t}{1+t^{2}}<\infty .
$$

Step 6. (iii) $\Longrightarrow$ (ii). Assume that $B=B_{\mathfrak{m}}\left(\mathcal{H}_{(a)}\right)$ with data $\mathfrak{m}, a$ as stated in (iii). Since $\mathfrak{m}^{b}(x) \leq \mathfrak{m}(x), x \in D$, and $\mathbb{R} \backslash D$ has measure zero, we have

$$
\int_{\mathbb{R}}\left(\log ^{+} \frac{\mathfrak{m}^{b}(t)}{|E(t)|}\right) \frac{d t}{1+t^{2}} \leq \int_{D}\left(\log ^{+} \frac{\mathfrak{m}(t)}{|E(t)|}\right) \frac{d t}{1+t^{2}}<\infty .
$$

Hence, the function $\mathfrak{m}_{B}^{\perp}$ is well defined and is an $\mathcal{N}_{+}$-majorant. What we showed at Step 4 applies to $\mathfrak{m}_{B}^{\perp}$. Relation (5.6) with $b=\operatorname{mt}_{\mathcal{H}} \mathfrak{m}_{B}^{\perp}=\mathrm{mt}_{\mathcal{H}} B$ gives

$$
B_{\mathfrak{m}_{\bar{B}}^{\perp}}(\mathcal{H})=B_{\left(\mathfrak{m}_{\bar{B}}^{\perp}\right)^{*}}\left(\mathcal{H}_{\left(\mathrm{mt}_{\mathcal{H}} B\right)}\right) \text {. }
$$

However, by Lemma $3.9(\mathrm{i})$, we have $\left(\mathfrak{m}_{B}^{\perp}\right)^{*}=\left.\mathfrak{m}^{\mathfrak{b}}\right|_{\mathbb{R}}$. Moreover, since the domain of $\mathfrak{m}$ is contained in $\mathbb{R}$, from [BW1, Theorem 3.4] it follows that $\operatorname{mt}_{\mathcal{H}_{(a)}} B=0$. Hence,

$$
\operatorname{mt}_{\mathcal{H}} B=\operatorname{mt}_{\mathcal{H}} \mathcal{H}_{(a)}=a,
$$

and we conclude that

$$
B=B_{\mathfrak{m}}\left(\mathcal{H}_{(a)}\right)=B_{\mathfrak{m}^{\mathrm{b}} \mid \mathbb{R}}\left(\mathcal{H}_{(a)}\right)=B_{\mathfrak{m}_{\bar{B}}^{\perp}}(\mathcal{H}) .
$$

Here, for the second equality, we have used Lemma 3.4.

Let us explicitly point out the following facts, which have been established in the course of the proof of Theorem 5.3. For a subset $B$ of the unit ball of $\mathcal{H}$, denote

$$
M^{\log }(B):=\left\{\mathfrak{m} \in \operatorname{Adm}_{\mathbb{C}^{+}} \mathcal{H}: \mathfrak{m} \log \text {-superharmonic, } B_{\mathfrak{m}}(\mathcal{H})=B\right\} .
$$

Corollary 5.4. Let $\mathcal{H}$ be a de Branges space, let $B \subseteq B(\mathcal{H}), B \neq \varnothing,\{0\}$, and assume that $M^{\log }(B) \neq \varnothing$. Then $\mathfrak{m}_{B}^{\perp}$ is well defined, belongs to $M^{\log }(B)$, and is the smallest element of this set. In particular, $M^{\log }(B)$ possesses a smallest element, and this element is an $\mathcal{N}_{+}$-majorant.

Proof. The fact that $\mathfrak{m}_{B}^{\perp}$ is well defined and belongs to $M^{\log }(B)$ was established at Steps 2 and 3 of the proof of Theorem 5.3 . Relation (5.4) shows that $\mathfrak{m}_{B}^{\perp} \leq \mathfrak{m}$ whenever $\mathfrak{m} \in M^{\log }(B)$, whence $\mathfrak{m}_{B}^{\perp}$ is the smallest element of this set.

Corollary 5.5. Let $\mathcal{H}$ be a de Branges space, and let $E \in \mathcal{H B}$ be such that $\mathcal{H}=\mathcal{H}(E)$. Then

$$
\begin{aligned}
& \left\{B_{\mathfrak{m}}(\mathcal{H}): \mathfrak{m} \in \operatorname{Adm}_{\mathbb{C}^{+}} \mathcal{H}, \mathfrak{m} \text { log-superharmonic }\right\} \\
& =\left\{\begin{array}{ll}
B_{\mathfrak{m}}\left(\mathcal{H}_{(a)}\right): \begin{array}{l}
a \leq 0, \mathfrak{m} \in \operatorname{Adm}_{\mathbb{R}} \mathcal{H}_{(a)}, \mathfrak{m} \text { continuous, }, \\
\int_{\mathbb{R}}\left(\log ^{+} \frac{\mathfrak{m}(t)}{|E(t)|}\right) \frac{d t}{1+t^{2}}<\infty
\end{array}
\end{array}\right\} .
\end{aligned}
$$

Proof. The inclusion " $\subseteq$ " follows from Theorem $[5.3$, (i) $\Longrightarrow$ (iv). The reverse inclusion follows from the implication (iii) $\Longrightarrow$ (i).

Corollary 5.6. Let $\mathcal{H}$ be a de Branges space, and let $\mathfrak{m} \in \operatorname{Adm}_{\mathbb{C}^{+}} \mathcal{H}$ be an $\mathcal{N}_{+}$-majorant. Then

$$
B_{\mathfrak{m}}(\mathcal{H})=B_{\mathfrak{m}^{*}}\left(\mathcal{H}_{\left(\mathrm{mt}_{\mathcal{H}} \mathfrak{m}\right)}\right) \quad \text { and } \quad \operatorname{mt}_{\mathcal{H}} B_{\mathfrak{m}}(\mathcal{H})=\mathrm{mt}_{\mathcal{H}} \mathfrak{m} .
$$

Proof. These assertions follow immediately from (5.6) and (5.8). 
In order to gain logical clarity, we wish to make the following point explicit.

Remark 5.7. In Lemma 4.1 and Corollary 4.5 we obtained examples of unit balls $B_{\mathfrak{m}}(\mathcal{H})$ invariant under division by Blaschke products. Theorem 5.3 implies that the set of unit balls described in (the almost trivial) Lemma 4.1 already includes the set of unit balls described by (the nontrivial) Corollary 4.5. However, this does not make Corollary 4.5 superfluous; it was essentially needed for the proof of Theorem 5.3 .

In this context, we note that the class given by Lemma 4.1 is generically even strictly larger than the class of balls generated by log-superharmonic majorants.

Example 5.8. Suppose $E \in \mathcal{H} B$ has no real zeros and assume that there exists a de Branges subspace $\mathcal{L}_{0}$ of $\mathcal{H}(E)$ that is not of the form $\mathcal{H}_{(a)}$. Consider the majorant

$$
\mathfrak{m}(x):=\nabla_{\mathcal{H}(E)}(x), \quad x \in \mathbb{R} .
$$

Then, for each subspace $\mathcal{L}$ of $\mathcal{H}(E)$ we have $B_{\mathfrak{m}}(\mathcal{L})=B(\mathcal{L})$. In particular, the ball $B_{\mathfrak{m}}\left(\mathcal{L}_{0}\right)$ is not of the form $B_{\mathfrak{m}}\left(\mathcal{H}_{(a)}\right)$ with some $a \leq 0$.

b. Condition (5.1). The first condition in Theorem 5.3 (iv) can be viewed as a geometric requirement, whereas the second condition, namely (5.1), is an analytic condition. In the present subsection we shall show that, generically, (5.1) plays only a minor role. Actually, it is satisfied in "most" cases. First let us find out when the integral condition in Corollary 5.5 is superfluous.

Lemma 5.9. Let $\mathcal{H}$ be a de Branges space, choose $E \in \mathcal{H} B$ with $\mathcal{H}=\mathcal{H}(E)$, and denote by $\varphi_{E}$ a phase function of $E$. Then

$$
\begin{aligned}
& \left\{B_{\mathfrak{m}}(\mathcal{H}): \mathfrak{m} \in \operatorname{Adm}_{\mathbb{C}^{+}} \mathcal{H}, \mathfrak{m} \log \text {-superharmonic }\right\} \\
& \quad=\left\{B_{\mathfrak{m}}\left(\mathcal{H}_{(a)}\right): a \leq 0, \mathfrak{m} \in \operatorname{Adm}_{\mathbb{R}} \mathcal{H}_{(a)}, \mathfrak{m} \text { continuous }\right\}
\end{aligned}
$$

if and only if

$$
\int_{\mathbb{R}}\left[\log ^{+} \varphi_{E}^{\prime}(t)\right] \frac{d t}{1+t^{2}}<\infty .
$$

Proof. By the formula for $\nabla_{\mathcal{H}}$ given in Subsection 2.9, we have $|E|^{-1} \nabla_{\mathcal{H}}=(\sqrt{\pi})^{-1} \sqrt{\varphi_{E}^{\prime}}$ along the real axis. Hence,

$$
\frac{1}{2} \log ^{+} \varphi_{E}^{\prime}(t)-\log \sqrt{\pi} \leq \log ^{+} \frac{\nabla_{\mathcal{H}}(t)}{|E(t)|} \leq \frac{1}{2} \log ^{+} \varphi_{E}^{\prime}(t), \quad t \in \mathbb{R}
$$

and we conclude that the convergence of the integral (5.9) is equivalent to

$$
\int_{\mathbb{R}}\left(\log ^{+} \frac{\nabla_{\mathcal{H}}(t)}{|E(t)|}\right) \frac{d t}{1+t^{2}}<\infty
$$

Assume that the stated equality of sets is valid. The unit ball of $\mathcal{H}$ can be written as $B(\mathcal{H})=B_{\nabla_{\mathcal{H}} \mid \mathbb{R}}(\mathcal{H})$. Since $\mathfrak{m}_{B(\mathcal{H})}=\nabla_{\mathcal{H}}$, the convergence of the integral in (5.10) follows from Theorem 5.3 .

Conversely, assume that (5.10) is true, and let a continuous majorant $\mathfrak{m} \in \operatorname{Adm}_{\mathbb{R}} \mathcal{H}_{(a)}$ be given. Since $B_{\mathfrak{m}}\left(\mathcal{H}_{(a)}\right)=B_{\mathfrak{m}^{b} \mid \mathbb{R}}\left(\mathcal{H}_{(a)}\right)$ and $\mathfrak{m}^{b} \leq \nabla_{\mathcal{H}}$, from Theorem 5.3 we see that $B_{\mathfrak{m}}\left(\mathcal{H}_{(a)}\right)$ can be represented with some log-superharmonic majorant. This proves the inclusion " $\supseteq$ " in the stated equality. The inclusion " $\subseteq$ " is fulfilled in any case.

In view of this fact, it is of interest to see that the convergence of the integral in (5.9) follows from a mild condition on the density of the zeros of $E$. 
Proposition 5.10. Let $E \in \mathcal{H B}$, and denote by $n(t)$ the counting function for the nonreal zeros of $E$; i.e., let $n(t)$ be the number of the nonreal zeros of $E$ located in the disk $\{z \in \mathbb{C}:|z| \leq t\}$, counted according to their multiplicities. If

$$
\int_{1}^{\infty} \frac{\log n(t)}{t^{2}} d t<\infty
$$

then (5.9) is true.

Proof. Denote by $z_{n}=x_{n}-i y_{n}, n=1,2, \ldots$, the (finite or infinite) sequence of nonreal zeros of $E$, and set $c:=-2^{-1} \operatorname{mt}\left(E^{-1} E^{\#}\right)$. Moreover, let $\varphi_{E}$ be a phase function of $E$. Then

$$
\sum_{n} \operatorname{Im} \frac{1}{z_{n}}=\sum_{n} \frac{y_{n}}{\left|z_{n}\right|^{2}}<\infty \quad \text { and } \quad \varphi_{E}^{\prime}(t)=c+\sum_{n} \frac{y_{n}}{\left|t-z_{n}\right|^{2}} .
$$

Let $k \in \mathbb{N}$ be fixed. Then, for $t \in[k-1, k]$ and $|z|>2 k$, we have

$$
\frac{|\operatorname{Im} z|}{|t-z|^{2}}=\frac{|\operatorname{Im} z|}{|z|^{2}} \cdot \frac{1}{\left|1-\frac{t}{z}\right|^{2}} \leq 2 \frac{|\operatorname{Im} z|}{|z|^{2}} .
$$

Thus,

$$
\sum_{\left|z_{n}\right|>2 k} \frac{y_{n}}{\left|t-z_{n}\right|^{2}} \leq 2 \sum_{\left|z_{n}\right|>2 k} \frac{y_{n}}{\left|z_{n}\right|^{2}} \leq 2 \sum_{n} \frac{y_{n}}{\left|z_{n}\right|^{2}} .
$$

Set $A:=1+c+2 \sum_{n}\left|z_{n}\right|^{-2} y_{n}$, then for $t \in[k-1, k]$ we have

$$
\begin{aligned}
\log ^{+} \varphi_{E}^{\prime}(t) \leq \log \left(1+\varphi_{E}^{\prime}(t)\right) & \leq \log \left(1+c+\sum_{\left|z_{n}\right|>2 k} \frac{y_{n}}{\left|t-z_{n}\right|^{2}}+\sum_{\left|z_{n}\right| \leq 2 k} \frac{y_{n}}{\left|t-z_{n}\right|^{2}}\right) \\
& \leq \log \left(A+\sum_{\left|z_{n}\right| \leq 2 k} \frac{y_{n}}{\left|t-z_{n}\right|^{2}}\right) .
\end{aligned}
$$

By the Jensen inequality,

$$
\begin{aligned}
\int_{k-1}^{k} \log ^{+} \varphi_{E}^{\prime}(t) d t & \leq \int_{k-1}^{k} \log \left(A+\sum_{\left|z_{n}\right| \leq 2 k} \frac{y_{n}}{\left|t-z_{n}\right|^{2}}\right) d t \\
& \leq \log \int_{k-1}^{k}\left(A+\sum_{\left|z_{n}\right| \leq 2 k} \frac{y_{n}}{\left|t-z_{n}\right|^{2}}\right) d t \leq \log (A+\pi n(2 k)) .
\end{aligned}
$$

Here we have used the fact that

$$
\int_{k-1}^{k} \frac{y_{n}}{\left|t-z_{n}\right|^{2}} d t \leq \int_{\mathbb{R}} \frac{y_{n}}{\left|t-z_{n}\right|^{2}} d t=\pi
$$

Now we can estimate

$$
\begin{aligned}
\int_{0}^{\infty}\left[\log ^{+} \varphi_{E}^{\prime}(t)\right] \frac{d t}{1+t^{2}} & =\sum_{k=1}^{\infty} \int_{k-1}^{k}\left[\log ^{+} \varphi_{E}^{\prime}(t)\right] \frac{d t}{1+t^{2}} \\
& \leq \sum_{k=1}^{\infty} \frac{1}{1+(k-1)^{2}} \int_{k-1}^{k} \log ^{+} \varphi_{E}^{\prime}(t) d t \leq \sum_{k=1}^{\infty} \frac{\log (A+\pi n(2 k))}{1+(k-1)^{2}} .
\end{aligned}
$$

Our hypothesis (5.11) implies that the last series converges. The integral

$$
\int_{-\infty}^{0}\left[\log ^{+} \varphi_{E}^{\prime}(t)\right]\left(1+t^{2}\right)^{-1} d t
$$

can be estimated in the same way, and we obtain (5.9). 
Corollary 5.11. If $E \in \mathcal{H B}$ is of finite order, then condition (5.11) holds true. Hence, for de Branges spaces $\mathcal{H}=\mathcal{H}(E)$, where $E$ is of finite order, the analytic condition in (5.1) is always satisfied.

Example 5.12. Although condition (5.11) is not necessary for (5.9), the following example shows that it is pretty sharp. Let

$$
z_{n}=\ln |n|+\frac{i}{|n| \ln ^{2}|n|}, \quad n \in \mathbb{Z}, \quad n \neq 0 .
$$

Then $c_{1} e^{t} \leq n(t) \leq c_{2} e^{t}$ with some constants $c_{1}, c_{2}>0$, and thus, the integral in (5.11) "just" diverges. Let $t \in[\ln k, \ln k+1], k>1$. Then

$$
\varphi_{E}^{\prime}(t)>\sum_{k / 2 \leq n \leq k-1} \frac{1}{n \ln ^{2} n\left[(\ln n-\ln k)^{2}+n^{-2} \ln ^{-4} n\right]} .
$$

Note that $\ln k-\ln n \asymp \frac{k-n}{n}>\frac{1}{n \ln ^{2} n}$. Consequently,

$$
\varphi_{E}^{\prime}(t)>C \sum_{k / 2 \leq n \leq k-1} \frac{n}{(k-n)^{2} \ln ^{2} n} \geq C_{1} \frac{k}{\ln ^{2} k} \geq C_{2} t^{-2} e^{t},
$$

and the log-integral diverges.

c. Hardy class majorants. Let $\mathcal{H}=\mathcal{H}(E)$ be a de Branges space, and let $\mathcal{L}=\mathcal{H}\left(E_{1}\right)$ be one of its de Branges subspaces. A standard majorant, which was already used in [BW1] and investigated in greater detail in [BW3], is the function

$$
\mathfrak{m}_{E_{1}}(z):=|z+i|^{-1}\left|E_{1}(z)\right|, \quad z \in \mathbb{C}^{+} \cup \mathbb{R} .
$$

This majorant reproduces the space $\mathcal{L}$ in the sense that $\mathcal{R}_{\mathfrak{m}_{E_{1}}}(\mathcal{H})=\mathcal{L}$. Since we can write

$$
\mathfrak{m}_{E_{1}}(z)=\left|e^{-i \operatorname{mt}\left[\left(E^{-1} E_{1}\right)\right] z} \cdot e^{i\left[\operatorname{mt}\left(E^{-1} E_{1}\right)\right] z} \frac{E_{1}(z)}{(z+i) E(z)} \cdot E(z)\right|, \quad z \in \mathbb{C}^{+},
$$

this function is an $\mathcal{N}_{+}$-majorant. Note that $E^{-1} E_{1}$ is outer for $\mathcal{N}$, because it has a continuous extension to $\mathbb{C}^{+} \cup \mathbb{R}$ that vanishes only on a discrete set and tends to zero at most polynomially; see [RR, Theorem 3.13] once again. However, the function

$$
f(z):=e^{i\left[\operatorname{mt}\left(E^{-1} E_{1}\right)\right] z} \frac{E_{1}(z)}{(z+i) E(z)}
$$

is not only outer for $\mathcal{N}$, but also actually outer for $H^{2}$.

Definition 5.13. Let $\mathcal{H}$ be a de Branges space, and choose $E \in \mathcal{H} B$ with $\mathcal{H}=\mathcal{H}(E)$. Moreover, let $p \in(0, \infty)$. A function $\mathfrak{m} \in \operatorname{Adm}_{\mathbb{C}^{+}} \mathcal{H}$ is called an $H^{p}$-majorant for $\mathcal{H}$ if it is of the form

$$
\mathfrak{m}(z)=\left|e^{-i a z} f(z) E(z)\right|, \quad z \in \mathbb{C}^{+},
$$

with some $a \leq 0$ and $f$ being outer for $H^{p}$.

Since $\mathcal{H}\left(E_{1}\right)=\mathcal{H}\left(E_{2}\right)$ implies not only that $E_{1}^{-1} E_{2}$ is outer, but also that this quotient is bounded from above and bounded away from zero throughout the half-plane $\mathbb{C}^{+}$, this notion does not depend on a particular choice of $E$ in Definition 5.13 .

For a subset $B$ of the unit ball of $\mathcal{H}$, denote

$$
M^{p}(B):=\left\{\mathfrak{m} \in \operatorname{Adm} \mathcal{H}: \mathfrak{m} \text { is an } H^{p} \text {-majorant, } B_{\mathfrak{m}}(\mathcal{H})=B\right\} .
$$

Clearly, $M^{p}(B) \subseteq M^{\log }(B)$. Now, an analog of Theorem 5.3 including Corollary 5.4 and corresponding to the set $M^{p}(B)$, reads as follows. 
Proposition 5.14. Let $\mathcal{H}$ be a de Branges space, let $B$ be a nonempty subset of its unit ball, and let $p \in(0, \infty)$. The following statements are equivalent.

(i) $M^{p}(B) \neq \varnothing$; i.e., there exists an $H^{p}$-majorant $\mathfrak{m} \in \operatorname{Adm}_{\mathbb{C}^{+}} \mathcal{H}$ such that $B=$ $B_{\mathfrak{m}}(\mathcal{H})$.

(ii) There exists a subset $D \subseteq \mathbb{R}$ such that $\mathbb{R} \backslash D$ has measure zero, a number a $\leq 0$, and a measurable majorant $\mathfrak{m} \in \operatorname{Adm}_{D} \mathcal{H}_{(a)}$ satisfying

$$
\int_{D}\left(\frac{\mathfrak{m}(t)}{|E(t)|}\right)^{p} d t<\infty
$$

such that $B=B_{\mathfrak{m}}\left(\mathcal{H}_{(a)}\right)$.

(iii) We have $B=B_{\left.\mathfrak{m}_{B}\right|_{\mathbb{R}}}\left(\mathcal{H}_{\left(\mathrm{mt}_{\mathcal{H}} B\right)}\right)$ and $\int_{\mathbb{R}}\left(\frac{\mathfrak{m}_{B}(t)}{|E(t)|}\right)^{p} d t<\infty$.

In this case, the set $M^{p}(B)$ contains the smallest element. Actually, the majorant $\mathfrak{m}_{B}^{\perp}$ is an $H^{p}$-majorant.

Proof. Let (i) be true, and let $\mathfrak{m} \in \operatorname{Adm}_{\mathbb{C}^{+}} \mathcal{H}$ be an $H^{p}$-majorant with $B=B_{\mathfrak{m}}(\mathcal{H})$. Since $\mathfrak{m}$ is, in particular, log-superharmonic, Theorem 5.3 yields $B=B_{\mathfrak{m}_{B} \mid \mathbb{R}}\left(\mathcal{H}_{\left(\mathrm{mt}_{\mathcal{H}} B\right)}\right)$. Let $\mathfrak{m}^{*}$ be the boundary function of $\mathfrak{m}$; cf. (5.5). Then $|E|^{-1} \mathfrak{m}^{*} \in L^{p}(d t)$, and from $\mathfrak{m}_{B} \preccurlyeq \mathfrak{m}^{*}$ we conclude that also $|E|^{-1} \mathfrak{m}_{B} \in L^{p}(d t)$.

The implication (iii) $\Longrightarrow$ (ii) is trivial. It remains to show that (ii) implies (i). For this, assume that $B=B_{\mathfrak{m}}\left(\mathcal{H}_{(a)}\right)$ with data $\mathfrak{m}, a$ as in (ii). Then, by Theorem 5.3 , $M^{\log }(B) \neq \varnothing$, and Corollary 5.4 shows that $B=B_{\mathfrak{m}_{B}^{\perp}}(\mathcal{H})$. Since $\mathfrak{m}_{B} \preccurlyeq \mathfrak{m}$, the convergence of the integral (5.13) implies that $\left.\left(|E|^{-1} \mathfrak{m}_{B}\right)\right|_{\mathbb{R}} \in L^{p}(d t)$. Therefore, the outer function $\mathfrak{f}_{|E|^{-1} \mathfrak{m}_{B}}$ used in the definition of $\mathfrak{m}_{B}^{\perp}$ is outer for $H^{p}$; i.e., $\mathfrak{m}_{B}^{\perp}$ is an $H^{p}$-majorant.

Let $\mathfrak{m}_{0} \in \operatorname{Adm} \mathcal{H}$, choose $E_{1} \in \mathcal{H B}$ with $\mathcal{R}_{\mathfrak{m}_{0}}(\mathcal{H})=\mathcal{H}\left(E_{1}\right)$, and let $\mathfrak{m}_{E_{1}}$ be the $H^{2}$-majorant defined in (5.12). Then $\mathcal{R}_{\mathfrak{m}_{0}}(\mathcal{H})=\mathcal{R}_{\mathfrak{m}_{E_{1}}}(\mathcal{H})$. But of course, in general, the balls $B_{\mathfrak{m}_{0}}(\mathcal{H})$ and $B_{\mathfrak{m}_{E_{1}}}(\mathcal{H})$ will by no means be comparable. The next statement shows that, at least as far as majorization along $\mathbb{R}$ is concerned, an $H^{2}$-majorant realizing $\mathcal{R}_{\mathfrak{m}_{0}}(\mathcal{H})$ can be chosen to be small.

Proposition 5.15. Let $a \leq 0$, and let $\mathfrak{m}_{0} \in \operatorname{Adm}_{\mathbb{R}} \mathcal{H}_{(a)}$ be given. Then there exists an $H^{2}$-majorant $\mathfrak{m} \in \operatorname{Adm} \mathcal{H}$ such that

$$
\mathcal{R}_{\mathfrak{m}}(\mathcal{H})=\mathcal{R}_{\mathfrak{m}_{0}}\left(\mathcal{H}_{(a)}\right) \quad \text { and } \quad B_{\mathfrak{m}}(\mathcal{H}) \subseteq B_{\mathfrak{m}_{0}}\left(\mathcal{H}_{(a)}\right) .
$$

Proof. Since $\mathcal{H}$ is a separable Hilbert space, we can choose a countable set $\left\{F_{n}: n \in \mathbb{N}\right\} \subseteq$ $B_{\mathfrak{m}_{0}}(\mathcal{H})$ dense in $B_{\mathfrak{m}_{0}}(\mathcal{H})$ in the norm of $\mathcal{H}$. We set $G_{n}:=2^{-n} F_{n}, n \in \mathbb{N}$, and define a continuous function $\mathfrak{m}_{1}: \mathbb{R} \rightarrow[0, \infty)$ by

$$
\mathfrak{m}_{1}(x):=\sup _{n \in \mathbb{N}}\left|G_{n}(x)\right|, \quad x \in \mathbb{R} .
$$

Continuity follows, because the functions $G_{n}, n \in \mathbb{N}$, belong to $B(\mathcal{H})$, and hence form an equicontinuous family; cf. Step 1 of the proof of Lemma 3.3.

We have $\mathfrak{m}_{1} \leq \mathfrak{m}_{0}$, whence

$$
B_{\mathfrak{m}_{1}}\left(\mathcal{H}_{(a)}\right) \subseteq B_{\mathfrak{m}_{0}}\left(\mathcal{H}_{(a)}\right) .
$$

Since $G_{n} \in B_{\mathfrak{m}_{1}}\left(\mathcal{H}_{(a)}\right)$ for each $n \in \mathbb{N}$, we see that $\mathfrak{m}_{1} \in \operatorname{Adm}_{\mathbb{R}} \mathcal{H}_{(a)}$, and actually $R_{\mathfrak{m}_{1}}\left(\mathcal{H}_{(a)}\right) \supseteq \operatorname{span}\left\{F_{n}: n \in \mathbb{N}\right\}$. This implies that $\mathcal{R}_{\mathfrak{m}_{1}}\left(\mathcal{H}_{(a)}\right) \supseteq \mathcal{R}_{\mathfrak{m}_{0}}\left(\mathcal{H}_{(a)}\right)$. However, by (5.14), the reverse inclusion also holds true, and it follows that

$$
\mathcal{R}_{\mathfrak{m}_{1}}\left(\mathcal{H}_{(a)}\right)=\mathcal{R}_{\mathfrak{m}_{0}}\left(\mathcal{H}_{(a)}\right) .
$$


Choose $E \in \mathcal{H} B$ with $\mathcal{H}=\mathcal{H}(E)$. Then

$$
\left(\int_{\mathbb{R}}\left|\frac{G_{n}(t)}{E(t)}\right|^{2} d t\right)^{\frac{1}{2}}=\left\|G_{n}\right\|_{\mathcal{H}}=\frac{1}{2^{n}}\left\|F_{n}\right\|_{\mathcal{H}} \leq \frac{1}{2^{n}},
$$

and therefore,

$$
\begin{aligned}
\int_{\mathbb{R}}\left(\frac{\mathfrak{m}_{1}(t)}{|E(t)|}\right)^{2} d t & =\int_{\mathbb{R}}\left[\sup _{n \in \mathbb{N}}\left|\frac{G_{n}(t)}{E(t)}\right|\right]^{2} d t \leq \int_{\mathbb{R}} \sum_{n=1}^{\infty}\left|\frac{G_{n}(t)}{E(t)}\right|^{2} d t \\
& =\sum_{n=1}^{\infty} \int_{\mathbb{R}}\left|\frac{G_{n}(t)}{E(t)}\right|^{2} d t \leq \sum_{n=1}^{\infty} \frac{1}{4^{n}}<\infty .
\end{aligned}
$$

Proposition 5.14 furnishes us with an $H^{2}$-majorant $\mathfrak{m} \in \operatorname{Adm}_{\mathbb{C}}+\mathcal{H}$ such that $B_{\mathfrak{m}}(\mathcal{H})=$ $B_{\mathfrak{m}_{1}}\left(\mathcal{H}_{(a)}\right)$. Using the fact that $\mathcal{H}_{(a)}$ is a closed subspace of $\mathcal{H}$, we obtain $\mathcal{R}_{\mathfrak{m}}(\mathcal{H})=$ $\mathcal{R}_{\mathfrak{m}_{1}}\left(\mathcal{H}_{(a)}\right)$.

\section{§6. Nonrepresentable Unit Balls}

Let $\mathfrak{m}_{0} \in \operatorname{Adm} \mathcal{H}$ and consider the unit ball $B_{\mathfrak{m}_{0}}(\mathcal{H})$. Then, in general, this ball need not be representable as $B_{\mathfrak{m}}(\mathcal{H})$ with some log-superharmonic majorant $\mathfrak{m}$; i.e., $M^{\log }\left(B_{\mathfrak{m}_{0}}(\mathcal{H})\right)$ can be empty. However, we may ask the question as to whether the ball $B_{\mathfrak{m}_{0}}(\mathcal{H})$ is contained in or contains some balls generated by such special kinds of majorants.

This question is also of interest for the following reason: in the first case, we obtain supersets $B$ such that division of a function $F$ in $B_{\mathfrak{m}_{0}}(\mathcal{H})$ by a Blaschke product cannot lead further out than $B$ and, in the second case, we obtain subsets $B$ that are invariant with respect to division by Blaschke products.

Definition 6.1. Let $\mathcal{H}$ be a de Branges space, and let $\mathfrak{m}_{0} \in \operatorname{Adm} \mathcal{H}$. Then we denote

$$
\begin{aligned}
& M_{\geq}^{\log }\left(\mathfrak{m}_{0}\right):=\left\{\mathfrak{m} \in \operatorname{Adm}_{\mathbb{C}^{+}} \mathcal{H}: \mathfrak{m} \text { log-superharmonic, } \mathfrak{m} \succcurlyeq \mathfrak{m}_{0}^{b}\right\} \\
& M_{\leq}^{\log }\left(\mathfrak{m}_{0}\right):=\left\{\mathfrak{m} \in \operatorname{Adm}_{\mathbb{C}^{+}} \mathcal{H}: \mathfrak{m} \text { log-superharmonic, } \mathfrak{m}^{b} \preccurlyeq \mathfrak{m}_{0}\right\}
\end{aligned}
$$

and

$$
B_{\geq}^{\log }\left(\mathfrak{m}_{0}\right):=\beta\left(M_{\geq}^{\log }\left(\mathfrak{m}_{0}\right)\right), \quad B_{\leq}^{\log }\left(\mathfrak{m}_{0}\right):=\beta\left(M_{\leq}^{\log }\left(\mathfrak{m}_{0}\right)\right),
$$

where $\beta$ is the map $\mathfrak{m} \mapsto B_{\mathfrak{m}}(\mathcal{H})$; cf. (2.4).

Observe that

$$
\begin{aligned}
& \mathfrak{m} \succcurlyeq \mathfrak{m}_{0}^{b} \Longleftrightarrow B_{\mathfrak{m}}(\mathcal{H}) \supseteq B_{\mathfrak{m}_{0}}(\mathcal{H}) \Longleftrightarrow \mathfrak{m}^{b} \geq \mathfrak{m}_{0}^{b}, \\
& \mathfrak{m}^{b} \preccurlyeq \mathfrak{m}_{0} \Longleftrightarrow B_{\mathfrak{m}}(\mathcal{H}) \subseteq B_{\mathfrak{m}_{0}}(\mathcal{H}) \Longleftrightarrow \mathfrak{m}^{b} \leq \mathfrak{m}_{0}^{b} .
\end{aligned}
$$

From the results of the previous section it will follow that the structure of the sets $M_{>}^{\log }\left(\mathfrak{m}_{0}\right)$ and $B_{\geq}^{\log }\left(\mathfrak{m}_{0}\right)$ is fairly simple. Contrasting this, we cannot say much about $M_{\leq}^{\log }\left(\mathfrak{m}_{0}\right)$ and $B_{\leq}^{\log }\left(\mathfrak{m}_{0}\right)$, an obvious obstruction being that in the definition of $M_{\leq}^{\log }\left(\mathfrak{m}_{0}\right)$ not the function $\mathfrak{m}$ itself but only $\mathfrak{m}^{b}$ occurs.

a. The set $M_{\geq}^{\log }\left(\mathfrak{m}_{0}\right)$. Whether or not a log-superharmonic majorant belongs to the set $M_{\geq}^{\log }(B)$ is decided by the behavior of boundary values along $\mathbb{R}$ and exponential growth.

Proposition 6.2. Let $\mathcal{H}$ be a de Branges space, and choose $E \in \mathcal{H B}$ with $\mathcal{H}=\mathcal{H}(E)$. If $\mathfrak{m}_{0} \in \operatorname{Adm} \mathcal{H}$, then the following statements hold true. 
(i) We have

$$
\begin{aligned}
& M_{\geq}^{\log }\left(\mathfrak{m}_{0}\right)=\left\{\mathfrak{m} \in \operatorname{Adm}_{\mathbb{C}^{+}} \mathcal{H}: \begin{array}{l}
\mathfrak{m} \text { log-superharmonic, and } \\
\left.\mathfrak{m}^{b}\right|_{\mathbb{R}} \geq\left.\mathfrak{m}_{0}^{b}\right|_{\mathbb{R}}, \operatorname{mt}_{\mathcal{H}} \mathfrak{m}^{b} \geq \operatorname{mt}_{\mathcal{H}} \mathfrak{m}_{0}^{b}
\end{array}\right\} \\
& =\left\{\mathfrak{m} \in \operatorname{Adm}_{\mathbb{C}^{+}} \mathcal{H}: \mathfrak{m} \log \right. \text {-superharmonic, and } \\
& \left.\liminf _{z \rightarrow x, z \in \mathbb{C}^{+}} \mathfrak{m}(z) \geq \mathfrak{m}_{0}^{b}(x), x \in \mathbb{R}, \quad \limsup _{|z| \rightarrow \infty, z \in \mathbb{C}^{+}} \frac{1}{\operatorname{Im} z} \log \frac{\mathfrak{m}_{0}^{b}(z)}{\mathfrak{m}(z)} \leq 0\right\}, \\
& B_{\geq}^{\log }\left(\mathfrak{m}_{0}\right)=\left\{B_{\mathfrak{m}}\left(\mathcal{H}_{(a)}\right): \int_{\mathbb{R}}\left(\log ^{+} \frac{\mathfrak{m}(t)}{|E(t)|}\right) \frac{d t}{1+t^{2}}<\infty, \mathfrak{m} \geq\left.\mathfrak{m}_{0}^{b}\right|_{\mathbb{R}}, a \geq \operatorname{mt}_{\mathcal{H}} \mathfrak{m}_{0}^{b}\right\} .
\end{aligned}
$$

(ii) Let $\mathfrak{m} \in \operatorname{Adm}_{\mathbb{C}^{+}} \mathcal{H}$ be an $\mathcal{N}_{+}$-majorant, and denote by $\mathfrak{m}^{*}$ its boundary function; cf. (5.5). Then

$$
\mathfrak{m} \in M_{\geq}^{\log }\left(\mathfrak{m}_{0}\right) \Longleftrightarrow \mathfrak{m}^{*} \succcurlyeq \mathfrak{m}_{0}^{b}, \mathrm{mt}_{\mathcal{H}} \mathfrak{m} \geq \mathrm{mt}_{\mathcal{H}} \mathfrak{m}_{0}^{b} .
$$

Proof. First, we assume that $\mathfrak{m} \in M_{\geq}^{\log }\left(\mathfrak{m}_{0}\right)$, then $\mathfrak{m}^{b} \geq \mathfrak{m}_{0}^{b}$, and hence, in particular,

$$
\left.\mathfrak{m}^{b}\right|_{\mathbb{R}} \geq\left.\mathfrak{m}_{0}^{b}\right|_{\mathbb{R}}, \quad \mathrm{mt}_{\mathcal{H}} \mathfrak{m}^{b} \geq \mathrm{mt}_{\mathcal{H}} \mathfrak{m}_{0}^{b} .
$$

Next, assume that $\mathfrak{m}$ is $\log$-superharmonic and that the above two inequalities hold true. Then $\mathfrak{m}_{B_{\mathfrak{m}}(\mathcal{H})}^{\perp} \geq \mathfrak{m}_{B_{\mathfrak{m}_{0}}(\mathcal{H})}^{\perp}$, whence $\mathfrak{m} \geq \mathfrak{m}_{B_{\mathfrak{m}}(\mathcal{H})}^{\perp} \geq \mathfrak{m}_{B_{\mathfrak{m}_{0}}(\mathcal{H})}^{\perp} \succcurlyeq \mathfrak{m}_{0}^{b}$. In particular,

$$
\liminf _{z \rightarrow x, z \in \mathbb{C}^{+}} \mathfrak{m}(z) \geq \mathfrak{m}_{0}^{b}(x), \quad x \in \mathbb{R}, \quad \limsup _{|z| \rightarrow \infty, z \in \mathbb{C}^{+}} \frac{1}{\operatorname{Im} z} \log \frac{\mathfrak{m}_{0}^{b}(z)}{\mathfrak{m}(z)} \leq 0 .
$$

We see that both inclusions " $\subseteq$ " in the first asserted line of equalities are fulfilled.

Assume that $\mathfrak{m}$ is log-superharmonic and satisfies (6.1). An application of the Phragmén-Lindelöf principle $[\mathbf{R R}$, Theorem 6.2] with the functions and sets

$$
u(z):=\log \mathfrak{m}_{0}^{b}-\log \mathfrak{m}, \quad h(z):=\operatorname{Im} z, \quad R:=\mathbb{R}, \quad S:=\{\infty\},
$$

shows that $\mathfrak{m}_{0}^{b}(z) \leq \mathfrak{m}(z)$ for all $z \in \mathbb{C}^{+}$. Thus, $\mathfrak{m} \in M_{\geq}^{\log }(\mathcal{H})$.

In order to see the stated form of $B_{\geq}^{\log }\left(\mathfrak{m}_{0}\right)$, in view of Corollary [5.5] it suffices to note that

$$
B_{\mathfrak{m}}\left(\mathcal{H}_{(a)}\right) \supseteq B_{\mathfrak{m}_{0}}(\mathcal{H}) \Longleftrightarrow a \geq \mathrm{mt}_{\mathcal{H}} B_{\mathfrak{m}_{0}}(\mathcal{H})=\mathrm{mt}_{\mathcal{H}} \mathfrak{m}_{0}^{b}, \mathfrak{m} \succcurlyeq \mathfrak{m}_{0}^{b} .
$$

Finally, assume that $\mathfrak{m}$ is an $\mathcal{N}_{+}$-majorant. Since $\mathfrak{m} \succcurlyeq \mathfrak{m}^{b}$ implies $\mathfrak{m}^{*} \succcurlyeq \mathfrak{m}^{b}$, the implication " $\Longrightarrow$ " in the asserted equivalence is immediate from what we already know about $M_{\geq}^{\log }\left(\mathfrak{m}_{0}\right)$. Now, assume that $\mathfrak{m}$ satisfies the conditions stated on the right-hand side. Then, by the definition of $\mathfrak{m}_{B_{\mathfrak{m}_{0}}(\mathcal{H})}^{\perp}$ and Lemma 3.9(ii), we have $\mathfrak{m} \geq \mathfrak{m}_{B_{\mathfrak{m}_{0}}(\mathcal{H})}^{\perp} \succcurlyeq \mathfrak{m}_{0}^{b}$.

Now it is easy to give an analog of Theorem 5.3 and Corollary 5.4 corresponding to the set $M_{\geq}^{\log }\left(\mathfrak{m}_{0}\right)$.

Theorem 6.3. Let $\mathcal{H}$ be a de Branges space and choose $E \in \mathcal{H B}$ with $\mathcal{H}=\mathcal{H}(E)$. Moreover, let $\mathfrak{m}_{0} \in \operatorname{Adm} \mathcal{H}$. Then

$$
M_{\geq}^{\log }\left(\mathfrak{m}_{0}\right) \neq \varnothing \Longleftrightarrow \int_{\mathbb{R}}\left(\log ^{+} \frac{\mathfrak{m}_{0}^{b}(t)}{|E(t)|}\right) \frac{d t}{1+t^{2}}<\infty .
$$

In this case the set $M_{\geq}^{\log }(B)$ contains the smallest element, namely, the $\mathcal{N}_{+}$-majorant $\mathfrak{m}_{B_{\mathfrak{m}_{0}}(\mathcal{H})}^{\perp}$. Moreover, $\beta\left(\mathfrak{m}_{B_{\mathfrak{m}_{0}}(\mathcal{H})}^{\perp}\right)$ is the smallest element of $B_{\geq}^{\log }\left(\mathfrak{m}_{0}\right)$. 
Proof. The implication " $\Longrightarrow$ " is obvious. Assume that the integral on the right-hand side of the asserted equivalence converges. Then the function $\mathfrak{m}_{B_{\mathfrak{m}_{0}}}^{\perp}(\mathcal{H})$ is well defined, is an $\mathcal{N}_{+}$-majorant, and satisfies

$$
\left(\mathfrak{m}_{B_{\mathfrak{m}_{0}}(\mathcal{H})}^{\perp}\right)^{*}=\left.\mathfrak{m}_{0}^{b}\right|_{\mathbb{R}}, \quad \operatorname{mt}_{\mathcal{H}} \mathfrak{m}_{B_{\mathfrak{m}_{0}}(\mathcal{H})}^{\perp}=\mathrm{mt}_{\mathcal{H}} \mathfrak{m}_{0}^{b}
$$

Thus, $\mathfrak{m}_{B_{\mathfrak{m}_{0}}(\mathcal{H})}^{\perp} \in M_{\geq}^{\log }\left(\mathfrak{m}_{0}\right)$. Let $\mathfrak{m} \in M_{\geq}^{\log }\left(\mathfrak{m}_{0}\right)$ be given. Then the $\mathcal{N}_{+}$-majorant $\mathfrak{m}_{B_{\mathfrak{m}}(\mathcal{H})}^{\perp}$ generates the same unit ball as $\mathfrak{m}$ does and satisfies $\mathfrak{m}_{B_{\mathfrak{m}}(\mathcal{H})}^{\perp} \leq \mathfrak{m}$; cf. Corollary [5.4. Hence, $\mathfrak{m}_{B_{\mathfrak{m}}(\mathcal{H})}^{\perp} \in M_{\geq}^{\log }\left(\mathfrak{m}_{0}\right)$, and it follows that

$$
\begin{aligned}
\left(\mathfrak{m}_{B_{\mathfrak{m}}(\mathcal{H})}^{\perp}\right)^{*} & \geq\left.\mathfrak{m}_{0}^{b}\right|_{\mathbb{R}}=\left(\mathfrak{m}_{B_{\mathfrak{m}_{0}}(\mathcal{H})}^{\perp}\right)^{*}, \\
\mathrm{mt}_{\mathcal{H}} \mathfrak{m}_{B_{\mathfrak{m}}(\mathcal{H})}^{\perp} & \geq \operatorname{mt}_{\mathcal{H}} \mathfrak{m}_{0}^{b}=\operatorname{mt}_{\mathcal{H}} \mathfrak{m}_{B_{\mathfrak{m}_{0}}(\mathcal{H})}^{\perp} .
\end{aligned}
$$

This implies that $\mathfrak{m} \geq \mathfrak{m}_{B_{\mathfrak{m}}(\mathcal{H})}^{\perp} \geq \mathfrak{m}_{B_{\mathfrak{m}_{0}}(\mathcal{H})}^{\perp}$.

Since $\beta$ is order-preserving and maps $M_{\geq}^{\log }\left(\mathfrak{m}_{0}\right)$ onto $B_{\geq}^{\log }\left(\mathfrak{m}_{0}\right)$, the image of the smallest element of $M_{\geq}^{\log }\left(\mathfrak{m}_{0}\right)$ is the smallest element in $B_{\geq}^{\log }\left(\mathfrak{m}_{0}\right)$.

Remark 6.4. The above statements concerning $M_{\geq}^{\log }\left(\mathfrak{m}_{0}\right)$ and $B_{\geq}^{\log }\left(\mathfrak{m}_{0}\right)$ have obvious analogs for arbitrary nonempty subsets $B$ of $B(\mathcal{H})$ in place of $B_{\mathfrak{m}_{0}}(\mathcal{\mathcal { H }})$. We should replace everywhere $\mathfrak{m}_{0}^{b}$ by $\mathfrak{m}_{B}$, and copy the above proofs word for word. We have decided to stick to the case $B=B_{\mathfrak{m}_{0}}(\mathcal{H})$, in order to stress the contrast between approximation from above and from below; compare Theorem 6.3 with Theorem 6.5 and Proposition 6.9 below.

b. The set $M_{\leq}^{\log }\left(\mathfrak{m}_{0}\right)$. Curiously, the structure of $M_{\leq}^{\log }\left(\mathfrak{m}_{0}\right)$ is much subtler and is related to a completely different topic, namely the existence of zero-free elements in $B_{\mathfrak{m}_{0}}(\mathcal{H})$.

Theorem 6.5. Let $\mathcal{H}$ be a de Branges space, and let $\mathfrak{m}_{0} \in \operatorname{Adm} \mathcal{H}$. Then the following statements are equivalent.

(i) $M_{\leq}^{\log }\left(\mathfrak{m}_{0}\right) \neq \varnothing$.

(ii) There exists an $H^{2}$-majorant $\mathfrak{m}$ with $\mathfrak{m} \leq\left.\mathfrak{m}_{0}^{b}\right|_{\mathbb{C}^{+}}$.

(iii) There exists an element $F \in B_{\mathfrak{m}_{0}}(\mathcal{H})$ that satisfies $F^{\#}=F$ and has no zeros in $\mathbb{C} \backslash \mathbb{R}$.

We formulate the crucial argument of the proof of this result separately.

Lemma 6.6. Let $\mathfrak{m} \in \operatorname{Adm} \mathcal{H}$ and assume that $R_{\mathfrak{m}}(\mathcal{H})$ is invariant with respect to division by Blaschke products. Then $B_{\mathfrak{m}}(\mathcal{H})$ contains a function $F$ with $F=F^{\#}$ that has no zeros off the real axis. In fact, whenever $G \in R_{\mathfrak{m}}(\mathcal{H}) \backslash\{0\}$, the choice of $F$ can be made so that $\left.\mathfrak{d}_{F}\right|_{\mathbb{R}} \geq\left.\mathfrak{d}_{G}\right|_{\mathbb{R}}$.

Proof. Let $G \in R_{\mathfrak{m}}(\mathcal{H}) \backslash\{0\}$ and set $F_{1}:=G+G^{\#}$ (in case $G=-G^{\#}$, use $F_{1}:=i\left(G-G^{\#}\right)$ instead). Then $F_{1} \in R_{\mathfrak{m}}(\mathcal{H}) \backslash\{0\}, F_{1}=F_{1}^{\#}$, and $\left.\mathfrak{d}_{F_{1}}\right|_{\mathbb{R}} \geq\left.\mathfrak{d}_{G}\right|_{\mathbb{R}}$.

If $F_{1}$ has no zeros in $\mathbb{C}^{+}$, then $F:=\left\|F_{1}\right\|_{\mathfrak{m}}^{-1} F_{1}$ has all the desired properties, and we are done. Otherwise, let $P$ be the Blaschke product for $\mathbb{C}^{+}$built with the zeros of $F_{1}$, and define

$$
F_{2}:=\frac{F_{1}}{P}+\left(\frac{F_{1}}{P}\right)^{\#}=F_{1}\left(\frac{1}{P}+P\right) .
$$

Clearly, $F_{2}=F_{2}^{\#}$ and $\left.\mathfrak{d}_{F_{2}}\right|_{\mathbb{R}} \geq\left.\mathfrak{d}_{F_{1}}\right|_{\mathbb{R}} \geq\left.\mathfrak{d}_{G}\right|_{\mathbb{R}}$.

Let $w \in \mathbb{C}^{+}$be given. If $F_{1}(w) \neq 0$, then $0<|P(w)|<1$. Hence, also $P(w)^{-1}+$ $P(w) \neq 0$, and we obtain $F_{2}(w) \neq 0$. In case $F_{1}(w)=0$, we have $F_{1}(w) P^{-1}(w) \neq 0$ and 
$\left(F_{1} P\right)(w)=0$, and again it follows that $F_{2}(w) \neq 0$. Setting $F:=\left\|F_{2}\right\|_{\mathfrak{m}}^{-1} F_{2}$, we obtain a function with all the required properties.

Proof of Theorem 6.5. First, we establish the implication (i) $\Longrightarrow$ (iii). Assume that $\mathfrak{m} \in M_{\leq}^{\log }\left(\mathfrak{m}_{0}\right)$. Then, by Corollary 4.5, the unit ball $B_{\mathfrak{m}}(\mathcal{H})$ is invariant with respect to division by Blaschke products, and hence, also $R_{\mathfrak{m}}(\mathcal{H})$ has this property. An application of Lemma 6.6 yields a function $F$ as required in (iii).

Next we show that (iii) $\Longrightarrow$ (ii). Let $F$ be as in (iii), and set $\mathfrak{m}(z):=|F(z)|, z \in \mathbb{C}^{+}$. Since $F$ is zero-free in $\mathbb{C}^{+}$, we have $\mathfrak{d}_{\mathfrak{m}}(w)=0, w \in \mathbb{C}^{+}$. Since $F$ is real, $F \in B_{\mathfrak{m}}(\mathcal{H})$. Moreover, $\mathfrak{m}(z)=\left|\left(E^{-1} F\right) \cdot E\right|$, whence $\mathfrak{m}$ is an $H^{2}$-majorant. Finally, since $F \in B_{\mathfrak{m}_{0}}(\mathcal{H})$, we have $\mathfrak{m} \leq\left.\mathfrak{m}_{0}^{b}\right|_{\mathbb{C}^{+}}$. Thus, $\mathfrak{m} \in M_{\leq}^{\log }\left(\mathfrak{m}_{0}\right)$.

The implication (ii) $\Longrightarrow$ (i) is trivial.

Just as we asked for minimal elements of $M_{\geq}^{\log }\left(\mathfrak{m}_{0}\right)$ and $B_{\geq}^{\log }\left(\mathfrak{m}_{0}\right)$, it is natural to seek for maximal elements $M_{\leq}^{\log }\left(\mathfrak{m}_{0}\right)$ or $B_{\leq}^{\log }\left(\mathfrak{m}_{0}\right)$. It will turn out, cf. item (iii) of Proposition 6.9 below, that, in general, such elements do not exist. However, a positive result can be obtained when restricting considerations to a specific subclass of $M_{\leq}^{\log }\left(\mathfrak{m}_{0}\right)$.

Definition 6.7. Let $\mathcal{H}$ be a de Branges space, let $\mathfrak{m}_{0} \in \operatorname{Adm} \mathcal{H}$, and denote by $D \subseteq$ $\mathbb{C}^{+} \cup \mathbb{R}$ the domain of $\mathfrak{m}_{0}$. Then we define

$$
\widetilde{M}_{\leq}^{\log }\left(\mathfrak{m}_{0}\right):=\left\{\mathfrak{m} \in M_{\leq}^{\log }\left(\mathfrak{m}_{0}\right): \mathfrak{m}(z) \leq \mathfrak{m}_{0}(z), z \in D \cap \mathbb{C}^{+}\right\},
$$

and set $\widetilde{B}_{\leq}^{\log }\left(\mathfrak{m}_{0}\right):=\beta\left(\widetilde{M}_{\leq}^{\log }\left(\mathfrak{m}_{0}\right)\right)$.

Of course, the additional requirement in the definition of $\widetilde{M}_{\leq}^{\log }\left(\mathfrak{m}_{0}\right)$ is a restriction only if $D \cap \mathbb{C}^{+} \neq \varnothing$. In case $D \subseteq \mathbb{R}$, we have $\widetilde{M}_{\leq}^{\log }\left(\mathfrak{m}_{0}\right)=M_{\leq}^{\log }\left(\mathfrak{m}_{0}\right)$, and hence also $\widetilde{B}_{\leq}^{\log }\left(\mathfrak{m}_{0}\right)=B_{\leq}^{\log }\left(\mathfrak{m}_{0}\right)$.

Note that, by the equivalence of (i) and (ii) in Theorem 6.5, we have in particular

$$
\widetilde{M}_{\leq}^{\log }\left(\mathfrak{m}_{0}\right) \neq \varnothing \quad \Longleftrightarrow M_{\leq}^{\log }\left(\mathfrak{m}_{0}\right) \neq \varnothing .
$$

Let us explicitly state the following observation.

Remark 6.8. Let $B$ be a nonempty subset of the unit ball of $\mathcal{H}$. Then $B \in \widetilde{B}_{\leq}^{\log }\left(\mathfrak{m}_{0}\right)$ if and only if the function $\mathfrak{m}_{B}^{\perp}$ is well defined and satisfies $\mathfrak{m}_{B}^{\perp}(z) \leq \mathfrak{m}_{0}(z), z \in D \cap \mathbb{C}^{+}$, and $B=\beta\left(\mathfrak{m}_{B}^{\perp}\right)$.

Proposition 6.9. Let $\mathcal{H}$ be a de Branges space, let $\mathfrak{m}_{0} \in \operatorname{Adm} \mathcal{H}$, and denote by $D$ the domain of $\mathfrak{m}_{0}$. Assume that $\widetilde{M}_{\leq}^{\log }\left(\mathfrak{m}_{0}\right) \neq \varnothing$. Then:

(i) if $D \subseteq \mathbb{C}^{+}$, then for each $\mathfrak{m} \in \widetilde{M}_{\leq}^{\log }\left(\mathfrak{m}_{0}\right)$ there exists a maximal element $\hat{\mathfrak{m}}$ of $\widetilde{M}_{\leq}^{\log }\left(\mathfrak{m}_{0}\right)$ with $\mathfrak{m} \leq \widehat{\mathfrak{m}}$

(ii) if $\bar{D} \cap \mathbb{C}^{+} \neq \varnothing$, then for each element $B \in \widetilde{B}_{\leq}^{\log }\left(\mathfrak{m}_{0}\right)$ there exists a maximal element $\widehat{B}$ of $\widetilde{B}_{\leq}^{\log }\left(\mathfrak{m}_{0}\right)$ with $B \subseteq \widehat{B}$

(iii) if $D \subseteq \mathbb{R}$ and $\int_{\mathbb{R}}\left[\log ^{+}\left(|E|^{-1} \mathfrak{m}_{0}^{b}\right)\right]\left(1+t^{2}\right)^{-1} d t<\infty$, then $B_{\leq}^{\log }\left(\mathfrak{m}_{0}\right)$ contains the largest element, namely, $B_{\mathfrak{m}_{0}}(\mathcal{H})$ itself;

(iv) if $D \subseteq \mathbb{R}$ and $\int_{\mathbb{R}}\left[\log ^{+}\left(|E|^{-1} \mathfrak{m}_{0}^{b}\right)\right]\left(1+t^{2}\right)^{-1} d t=\infty$, then no element of $B_{\leq}^{\log }\left(\mathfrak{m}_{0}\right)$ is maximal in this set.

Proof. Step 1. The case where $D \subseteq \mathbb{C}^{+}$. We establish the hypothesis of Zorn's lemma for the set $\widetilde{M}_{\leq}^{\log }\left(\mathfrak{m}_{0}\right)$. Let an ascending chain $\mathfrak{M}$ of elements of $\widetilde{M}_{\leq}^{\log }\left(\mathfrak{m}_{0}\right)$ be given, and set

$$
\widetilde{\mathfrak{m}}:=\sup _{\mathfrak{m} \in \mathfrak{M}} \mathfrak{m}
$$


Since $\left.\mathfrak{m}\right|_{D} \leq \mathfrak{m}_{0}, \mathfrak{m} \in \mathfrak{M}$, we also have $\left.\tilde{\mathfrak{m}}\right|_{D} \leq \mathfrak{m}_{0}$. Note that the domain of $\tilde{\mathfrak{m}}$, i.e., $\mathbb{C}^{+}$, entirely contains the domain of $\mathfrak{m}_{0}$, i.e., $D$. We conclude that $B_{\widetilde{\mathfrak{m}}}(\mathcal{H}) \subseteq B_{\mathfrak{m}_{0}}(\mathcal{H})$.

The family $(\log \mathfrak{m})_{\mathfrak{m} \in \mathfrak{M}}$ is a nondecreasing net of superharmonic functions. Pick an element $\mathfrak{m}_{1} \in \mathfrak{M}$. Then there exists a function $F \in B_{\mathfrak{m}_{1}}(\mathcal{H})$ that has no zeros in $\mathbb{C}^{+}$. Hence, $\log |F|$ is harmonic in $\mathbb{C}^{+}$and satisfies $\log |F| \leq \log \mathfrak{m}_{1}$. Corollary 2.15 implies that $\log \tilde{\mathfrak{m}}$ is superharmonic. We conclude that $\tilde{\mathfrak{m}} \in \widetilde{M}_{\leq}^{\log }\left(\mathfrak{m}_{0}\right)$; we have found an upper bound of $\mathfrak{M}$.

Step 2. The case where $D \cap \mathbb{C}^{+} \neq \varnothing$. Let $\mathfrak{B}$ be an ascending chain in $B_{\leq}^{\log }\left(\mathfrak{m}_{0}\right)$. For each $B \in \mathfrak{B}$, the majorant $\mathfrak{m}_{B}^{\perp}$ is well defined and belongs to $M_{\leq}^{\log }\left(\mathfrak{m}_{0}\right)$, and we have $B=\beta\left(\mathfrak{m}_{B}^{\perp}\right)$. Set

$$
\widetilde{\mathfrak{m}}:=\sup _{B \in \mathfrak{B}} \mathfrak{m}_{B}^{\perp}
$$

and

$$
k_{B}:=\left.\frac{\mathfrak{m}_{B}}{|E|}\right|_{\mathbb{R}}, \quad k:=\sup _{B \in \mathfrak{B}} k_{B}, \quad a:=\sup _{B \in \mathfrak{B}} \operatorname{mt}_{\mathcal{H}} B .
$$

First, we are going to show that

$$
\tilde{\mathfrak{m}}(z)=\left|e^{-i a z} \mathfrak{f}_{k}(z) E(z)\right|, \quad z \in \mathbb{C}^{+} .
$$

To this end, we verify the hypothesis of Corollary 2.16 for the family $\left(k_{B}\right)_{B \in \mathfrak{B} \text {. By }}$ Lemma 3.3, the function $k_{B}$ is continuous. The fact that $B_{1} \subseteq B_{2}$ implies $k_{B_{1}} \leq k_{B_{2}}$ is clear, and the condition $\log k_{B} \in L^{1}\left(\frac{d t}{1+t^{2}}\right)$ is simply the fact that $\mathfrak{m}_{B}^{\perp}$ is well defined.

It remains to establish the second condition in (2.7). Here we employ our assumption that $D \cap \mathbb{C}^{+} \neq \varnothing$. Choose $z_{0}=x_{0}+i y_{0} \in D \cap \mathbb{C}^{+}$, and let $c>0$ be such that

$$
c \frac{1}{1+t^{2}} \leq \frac{y_{0}}{\left(t-x_{0}\right)^{2}+y_{0}^{2}}, \quad t \in \mathbb{R} .
$$

Moreover, fix $B_{0} \in \mathfrak{B}$. Then, for each $B \in \mathfrak{B}$ with $B \supseteq B_{0}$,

$$
\begin{aligned}
& c\left(\int_{\mathbb{R}}\left[\log k_{B}(t)\right] \frac{d t}{1+t^{2}}-\int_{\mathbb{R}}\left[\log k_{B_{0}}(t)\right] \frac{d t}{1+t^{2}}\right) \\
& \quad \leq \int_{\mathbb{R}}\left[\log k_{B}(t)-\log k_{B_{0}}(t)\right] \frac{y_{0}}{\left(t-x_{0}\right)^{2}+y_{0}^{2}} d t=\pi \log \left|\frac{\mathfrak{f}_{k_{B}}\left(z_{0}\right)}{\mathfrak{f}_{k_{B_{0}}}\left(z_{0}\right)}\right|, \\
& \left|\mathfrak{f}_{k_{B}}\left(z_{0}\right)\right|=e^{-y_{0} \operatorname{mt}_{\mathcal{H}} B} \frac{\mathfrak{m}_{B}^{\perp}\left(z_{0}\right)}{\left|E\left(z_{0}\right)\right|} \leq e^{-y_{0} \operatorname{mt}_{\mathcal{H}} B_{0}} \frac{\mathfrak{m}_{0}\left(z_{0}\right)}{\left|E\left(z_{0}\right)\right|}, \\
& \int_{\mathbb{R}}\left[\log ^{-} k_{B}(t)\right] \frac{d t}{1+t^{2}} \leq \int_{\mathbb{R}}\left[\log ^{-} k_{B_{0}}(t)\right] \frac{d t}{1+t^{2}} .
\end{aligned}
$$

Putting together these estimates yields

$$
\begin{aligned}
& \int_{\mathbb{R}}\left[\log ^{+} k_{B}(t)\right] \frac{d t}{1+t^{2}}=\int_{\mathbb{R}}\left[\log k_{B}(t)\right] \frac{d t}{1+t^{2}}+\int_{\mathbb{R}}\left[\log ^{-} k_{B}(t)\right] \frac{d t}{1+t^{2}} \\
& \leq \frac{\pi}{c}\left(\log \mathfrak{m}_{0}\left(z_{0}\right)-y_{0} \mathrm{mt}_{\mathcal{H}} B_{0}\right.\left.-\log \left|E\left(z_{0}\right)\right|-\log \left|\mathfrak{f}_{k_{B_{0}}}\left(z_{0}\right)\right|\right) \\
&+2 \int_{\mathbb{R}}\left|\log k_{B}(t)\right| \frac{d t}{1+t^{2}}=: C<\infty .
\end{aligned}
$$

Hence, by monotonicity,

$$
\sup _{B \in \mathfrak{B}} \int_{\mathbb{R}}\left[\log ^{+} k_{B}(t)\right] \frac{d t}{1+t^{2}}=\sup _{\substack{B \in \mathfrak{B} \\ B \supseteq B_{0}}} \int_{\mathbb{R}}\left[\log ^{+} k_{B}(t)\right] \frac{d t}{1+t^{2}} \leq C .
$$

An application of Corollary 2.16 gives $\mathfrak{f}_{k}(z)=\lim _{B \in \mathfrak{B}} \mathfrak{f}_{k_{B}}(z), z \in \mathbb{C}^{+}$, and (6.2) follows. 
Next, we are going to show that $\tilde{\mathfrak{m}} \in \widetilde{M}_{\leq}^{\log }\left(\mathfrak{m}_{0}\right)$. Clearly, $\tilde{\mathfrak{m}}$ is an $\mathcal{N}_{+}$-majorant and satisfies

$$
\tilde{\mathfrak{m}}(z) \leq \mathfrak{m}_{0}(z), \quad z \in D \cap \mathbb{C}^{+} .
$$

Since for each $B \in \mathfrak{B}$ we have $B \subseteq B_{\mathfrak{m}_{0}}(\mathcal{H})$, it follows that

$$
\exp \left(k_{B}(x)\right)|E(x)|=\mathfrak{m}_{B}(x) \leq \mathfrak{m}_{0}^{b}(x), \quad x \in \mathbb{R} .
$$

Thus, also $\exp (k(x))|E(x)| \leq \mathfrak{m}_{0}^{b}(x), x \in \mathbb{R}$. Let $F \in B_{\widetilde{\mathfrak{m}}}(\mathcal{H})$ be given. Then, for each Lebesgue point of the function $\log k$, we have

$$
|F(x)|=\lim _{z \rightarrow x}|F(z)| \leq \lim _{z \rightarrow x} \tilde{\mathfrak{m}}(z)=\exp (k(x))|E(x)| \leq \mathfrak{m}_{0}^{b}(x) .
$$

By continuity and the fact that the set of Lebesgue points of $\log k$ is dense in $\mathbb{R}$, it follows that

$$
|F(x)| \leq \mathfrak{m}_{0}^{b}(x), \quad x \in \mathbb{R} .
$$

In particular, $|F(x)| \leq \mathfrak{m}_{0}(x), x \in D \cap \mathbb{R}$. Together with (6.3), this shows that $F \in$ $B_{\mathfrak{m}_{0}}(\mathcal{H})$. We conclude that, indeed, $\widetilde{\mathfrak{m}} \in \widetilde{M}_{\leq}^{\log }(\mathcal{H})$. Moreover, clearly, $\beta(\widetilde{\mathfrak{m}})$ is an upper bound of $\mathfrak{B}$.

Step 3. The case where $D \subseteq \mathbb{R}$. If the integral $\int_{\mathbb{R}}\left[\log \left(|E|^{-1} \mathfrak{m}_{0}^{b}\right)\right]\left(1+t^{2}\right)^{-1} d t$ converges, then $B_{\mathfrak{m}_{0}}(\mathcal{H}) \in B_{\leq}^{\log }\left(\mathfrak{m}_{0}\right)$ by Theorem [5.3. Trivially, it is the largest element of this set.

Assume that the above logarithmic integral diverges. If $B \in B_{\leq}^{\log }\left(\mathfrak{m}_{0}\right)$, then $\mathfrak{m}_{B} \leq \mathfrak{m}_{0}^{b}$ and $\int_{\mathbb{R}}\left[\log \left(|E|^{-1} \mathfrak{m}_{B}\right)\right]\left(1+t^{2}\right)^{-1} d t<\infty$. Hence, we cannot have $\mathfrak{m}_{B}=\mathfrak{m}_{0}^{b}$. Choose $x_{0} \in \mathbb{R}$ such that $\mathfrak{m}_{B}\left(x_{0}\right)<\mathfrak{m}_{0}^{b}\left(x_{0}\right)$, and let $F \in B_{\mathfrak{m}_{0}}(\mathcal{H})$ be such that $\mathfrak{m}_{B}\left(x_{0}\right)<\left|F\left(x_{0}\right)\right|$. Set

$$
\mathfrak{m}_{1}(x):=\max \left\{|F(x)|, \mathfrak{m}_{B}(x)\right\}, \quad x \in \mathbb{R} ;
$$

then $\mathfrak{m}_{B} \preccurlyeq \mathfrak{m}_{1} \preccurlyeq \mathfrak{m}_{0}$. However, $F \in B_{\mathfrak{m}_{1}}(\mathcal{H}) \backslash B$, whence

$$
B \subsetneq B_{\mathfrak{m}_{1}}(\mathcal{H}) \subseteq B_{\mathfrak{m}_{0}}(\mathcal{H}) .
$$

Moreover, since $E^{-1} F \in H^{2}$, it follows that

$$
\int_{\mathbb{R}}\left(\log ^{+} \frac{\mathfrak{m}_{1}(t)}{|E(t)|}\right) \frac{d t}{1+t^{2}} \leq \int_{\mathbb{R}}\left(\log ^{+}\left|\frac{F(t)}{E(t)}\right|\right) \frac{d t}{1+t^{2}}+\int_{\mathbb{R}}\left(\log ^{+} \frac{\mathfrak{m}_{B}(t)}{|E(t)|}\right) \frac{d t}{1+t^{2}}<\infty .
$$

Now, Theorem 5.3 shows that $B_{\mathfrak{m}_{1}}(\mathcal{H}) \in B_{\leq}^{\log }\left(\mathfrak{m}_{0}\right)$.

\section{REFERENCES}

[B] A. D. Baranov, Polynomials in the de Branges spaces of entire functions, Ark. Mat. 44 (2006), 16-38. MR 2237209 (2007m:46036)

[BW1] A. Baranov and H. Woracek, Subspaces of de Branges spaces generated by majorants, Canad. J. Math. 61 (2009), no. 3, 503-517. MR.2514481

[BW2] _ Finite-dimensional de Branges subspaces generated by majorants, Oper. Theory Adv. Appl., vol. 188, Birkhäuser Verlag, Basel, 2009, pp. 37-48. MR.2641246

[BW3] , Majorization in de Branges spaces. I. Representability of subspaces, J. Funct. Anal. 258 (2010), no. 8, 2601-2636. MR.2593335

[BW4] - Majorization in de Branges spaces. II. Banach spaces generated by majorants, arXiv:0906.2943v1 [math. CV], to appear in Collectanea Mathematica.

[BM] A. Beurling and P. Malliavin, On Fourier transforms of measures with compact support, Acta Math. 107 (1962), 291-309. MR.0147848 (26:5361)

[Bo] R. Boas, Entire functions, Acad. Press, New York, 1954. MR0068627 (16:914f)

[dB1] L. de Branges, Some Hilbert spaces of entire functions, Proc. Amer. Math. Soc. 10 (1959), 840-846. MR0114002 (22:4833)

[dB2] , Some Hilbert spaces of entire functions, Trans. Amer. Math. Soc. 96 (1960), 259-295. MR0133455 (14:A3289a) 
[dB3] - Some Hilbert spaces of entire functions. II, Trans. Amer. Math. Soc. 99 (1961), 118-152. MR0133456 (24:A3289b)

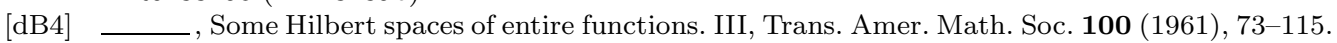
MR0133457 (24:A3289c)

[dB5] Some Hilbert spaces of entire functions. IV, Trans. Amer. Math. Soc. 105 (1962), 43-83. MR0143016 (26:583)

[dB6] , Hilbert spaces of entire functions, Prentice-Hall, Inc., Englewood Cliffs, NJ, 1968. MR0229011 (37:4590)

[HM1] V. P. Havin and J. Mashreghi, Admissible majorants for model subspaces of $H^{2}$. I. Slow winding of the generating inner function, Canad. J. Math. 55 (2003), no. 6, 1231-1263. MR2016246 (2004i:30029a)

[HM2] , Admissible majorants for model subspaces of $H^{2}$. II. Fast winding of the generating inner function, Canad. J. Math. 55 (2003), no. 6, 1264-1301. MR2016247(2004i:30029b)

[H-J] J. Hoffmann-Jørgensen, Stochastic processes on Polish spaces, Various Publ. Ser. (Aarhus), No. 39, Åarhus Univ., Mat. Inst., Aarhus, 1991. MR1217966 (95a:60047)

[KW] M. Kaltenbäck and H. Woracek, De Branges spaces of exponential type: general theory of growth, Acta Sci. Math. (Szeged) 71 (2005), no. 1-2, 231-284. MR2160366 (2006c:30031)

[RR] M. Rosenblum and J. Rovnyak, Topics in Hardy classes and univalent functions, Birkhäuser Verlag, Basel, 1994. MR.1307384 (97a:30047)

[R] Rudin W., Real and complex analysis, McGraw-Hill, New York, 1987. MR0924157 (88k:00002)

Department of Mathematics and Mechanics, St. Petersburg State University, Universitetskil Prospekt 28, Staryı̆ Peterhof, St. Petersburg 198504, Russia

E-mail address: a.baranov@ev13934.spb.edu

Institut für Analysis und Scientific Computing, Technische Universität Wien, Wiedner Hauptstrasse 8-10/101, A-1040 Wien, Austria

E-mail address: harald.woracek@tuwien.ac.at

Received 22/SEP/2009

Originally published in English 